\title{
Immunogenic ferroptosis and where to find it?
}

\author{
Robin Demuynck (D) , ,2 Iuliia Efimova (D) , , ${ }^{1,2}$ Faye Naessens, ${ }^{1}$ \\ Dmitri V Krysko (iD) 1,2,3,4
}

To cite: Demuynck R, Efimova I, Naessens F, et al. Immunogenic ferroptosis and where to find it? Journal for ImmunoTherapy of Cancer 2021;9:e003430. doi:10.1136/jitc-2021-003430

$\mathrm{RD}$ and IE contributed equally. Accepted 04 November 2021
Check for updates

(C) Author(s) (or their employer(s)) 2021. Re-use permitted under CC BY-NC. No commercial re-use. See rights and permissions. Published by BMJ.

${ }^{1}$ Cell Death Investigation and Therapy Lab, Department of Human Structure and Repair, Ghent University, Ghent, Belgium ${ }^{2}$ Cancer Research Institute Ghent, Ghent, Belgium

${ }^{3}$ Department of Pathophysiology, I M Sechenov First Moscow State Medical University, Moskva, Russian Federation ${ }^{4}$ Institute of Biology and Biomedicine, National Research Lobachevsky State University of Nizhny Novgorod, Niznij Novgorod, Russian Federation

Correspondence to Professor Dmitri V Krysko; dmitri.krysko@ugent.be

\section{ABSTRACT}

Ferroptosis is a recently discovered form of regulated cell death that is morphologically, genetically, and biochemically distinct from apoptosis and necroptosis, and its potential use in anticancer therapy is emerging. The strong immunogenicity of (early) ferroptotic cancer cells broadens the current concept of immunogenic cell death and opens up new possibilities for cancer treatment. In particular, induction of immunogenic ferroptosis could be beneficial for patients with cancers resistant to apoptosis and necroptosis. However, ferroptotic cancer cells may be a rich source of oxidized lipids, which contribute to decreased phagocytosis and antigen cross-presentation by dendritic cells and thus may favor tumor evasion. This could explain the non-immunogenicity of late ferroptotic cells. Besides the presence of lactate in the tumor microenvironment, acidification and hypoxia are essential factors promoting ferroptosis resistance and affecting its immunogenicity. Here, we critically discuss the crucial mediators controlling the immunogenicity of ferroptosis that modulate the induction of antitumor immunity. We emphasize that it will be necessary to also identify the tolerogenic (ie, immunosuppressive) nature of ferroptosis, which can lead to tumor evasion.

\section{CHARACTERISTICS OF FERROPTOSIS}

\section{Molecular mechanism of ferroptosis in brief}

Ferroptosis is a recently discovered form of regulated (necrotic) cell death. Since its discovery in 2012, it has rapidly become recognized as a potential anticancer strategy. ${ }^{1}$ Ferroptosis is iron-dependent and can be induced by blocking the system $\mathrm{X}_{\mathrm{C}}{ }^{-}$cystine/ glutamate antiporter or glutathione peroxidase 4 (GPX4), ${ }^{2}$ resulting in a defective glutathione (GSH) redox system, where oxidized phosphatidylethanolamines (oxPEs) are crucial for ferroptosis execution (figure 1, treatment examples in table 1). ${ }^{3}{ }^{34}$ More specifically, arachidonoyl and adrenoyl groups are oxidized in the endoplasmatic reticulum (ER)-associated compartments. ${ }^{5}$ Lipoxygenases (LOXs) oxidize those phosphatidylethanolamine (PE) species to cause cell death, and this can be blocked by tocopherols such as vitamin E ( $\alpha$-tocopherol). oxPEs cause an increase in membrane curvature that eventually leads to membrane destabilization, pore and micelle formation, and membrane rupture. ${ }^{6}$ Normally, glutamate is exchanged by cystine, which is then converted into cysteine, which is needed to produce GSH, an essential cofactor for GPX4. By blocking the $\mathrm{X}_{\mathrm{C}}{ }^{-}$antiporter, not enough cystine is transported into the cell, leading to a decrease in the available $\mathrm{GSH}^{7}$ When the pool of GSH is depleted, GPX4 can no longer function and lipid peroxidation can no longer be inhibited, leading to permeabilization of the cell membrane (figure 1). Alternatively, ferroptosis can be induced by directly blocking GPX4, which might result in a faster induction of ferroptotic cell death, as the GSH pool does not need to be depleted before the membrane is permeabilized.

Notably, iron has a considerable effect on lipid peroxidation in ferroptosis through the production of reactive oxygen species (ROS) by Fenton chemistry. ${ }^{8}$ Under physiological conditions, iron exists as ferric ions $\left(\mathrm{Fe}^{3+}\right)$, which can bind to transferrin and enter cells via the transferrin receptor (figure 1). Once in endosomes, ferric ions are reduced to ferrous ions $\left(\mathrm{Fe}^{2+}\right)$ in a reaction catalyzed by the ferric reductase STEAP3. $\mathrm{Fe}^{2+}$ is then transported through the endosomal membrane, with help from the divalent metal transporter 1 , into the cytosol, creating a labile iron pool (figure 1). Excess iron is either transported outside the cell by ferroportin, an iron exporter, or stored in ferritin protein, which oxidizes ferrous ions back to ferric ions. $\mathrm{Fe}^{2+}$ present in the labile iron pool can interact with $\mathrm{H}_{2} \mathrm{O}_{9}$, resulting in oxidation of $\mathrm{Fe}^{2+}$ back to $\mathrm{Fe}^{3+}$ and formation of highly reactive hydroxyl radicals $(\mathrm{OH} \bullet)$. $\mathrm{OH} \bullet$ then reacts with polyunsaturated fatty acids (PUFAs) of phospholipids (PLs) in the cell membrane, converting PL into carbon-centered PL radicals (PL $\bullet$ ). With the involvement of molecular oxygen $\left(\mathrm{O}_{2}\right)$ and adjacent PUFA, the primary lipid radical $(\mathrm{L} \bullet)$ is converted into an alkoxyl radical $(\mathrm{LO} \bullet)$, which can interact with $\mathrm{Fe}^{2+}$ and introduce a next round of lipid radical 

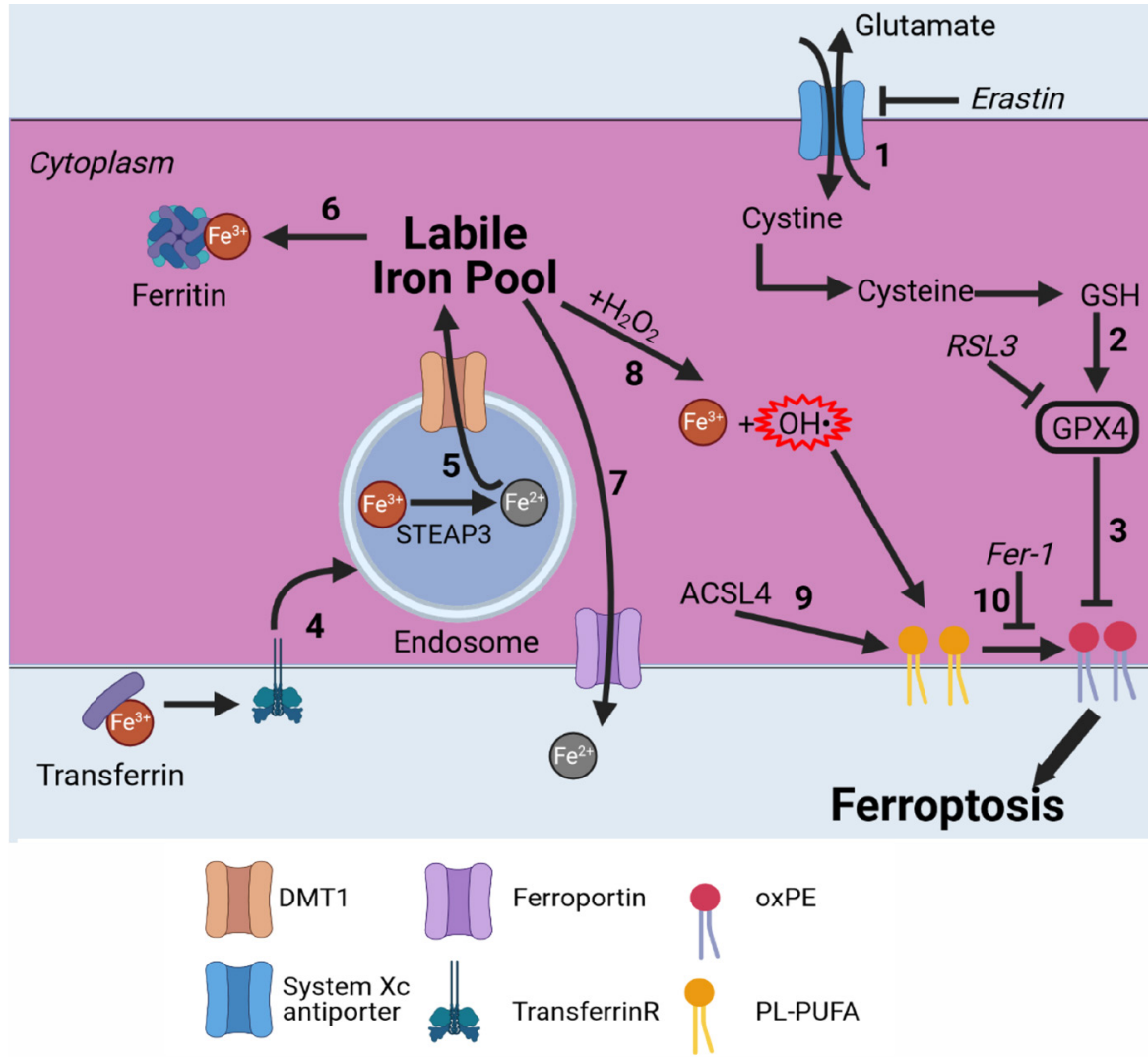

Figure 1 Ferroptosis pathway in brief. Ferroptosis is an iron-dependent cell death modality. (1) Cystine is imported into the cell in exchange for glutamate by the system $\mathrm{X}_{\mathrm{C}}^{-}$antiporter. Cystine is converted into cysteine and used for GSH production. (2) GSH is an essential cofactor for GPX4. By blocking the system $\mathrm{X}_{{ }^{-}}$antiporter (eg, with erastin), GSH is depleted and GPX4 can no longer function. (3) GPX4 inhibits lipid peroxidation; directly inhibiting it (eg, by RSL3) induces ferroptosis. (4) Fe ${ }^{3+}$ bound to transferrin is transported into endosomes by binding to the transferrin receptor. (5) $\mathrm{Fe}^{3+}$ is converted to Fe $\mathrm{Fe}^{2+}$ bTEAP3. Fe ${ }^{2+}$ is then transported to the labile iron pool by DMT1. (6) $\mathrm{Fe}^{2+}$ can be stored by binding to ferritin. (7) Fe $\mathrm{Fe}^{2+}$ can be transported outside the cell by ferroportin. (8) $\mathrm{Fe}^{2+}$ is oxidized back to $\mathrm{Fe}^{3+}$ via the Fenton reaction, ${ }^{8} 9$ leading to production of hydroxyl radicals. (9) ACSI4 selectively enriches PUFAs in the plasma membrane. (10) Radicals formed from the Fenton reaction react with PUFAs, leading to oxPE formation and cell death. This can be inhibited by Fer-1. ${ }^{10}$ ACSI4, acyl-CoA synthetase long-chain family member 4; CoA, coenzyme A; DMT1, divalent metal transporter 1; Fer-1, ferrostatin-1; GSH, glutathione; GPX4, glutathione peroxidase 4; oxPE, oxidized phosphatidylethanolamine; PUFA, polyunsaturated fatty acid; RSL3, Ras-selective lethal 3.

formation. Extensive production of lipid ROS promotes damage and/or destruction of the cell membrane, leading to cell death (figure 1). Several cell death inhibitors specific for ferroptosis have been developed. Ferrostatin-1 (Fer-1) can effectively block lipid peroxidation via a dual mechanism: on one hand, Fer-1 functions as a lipophilic radical scavenger reducing ROS formation, and on the other hand, it also blocks the 15-lipoxygenase (15LOX)/phosphatidylethanolamine-binding protein-1 (PEBP1) complex. ${ }^{10}$ This $15 \mathrm{LOX} / \mathrm{PEBP} 1$ complex is responsible, together with the Fe-catalyzed radical reaction, for oxPEs formation, meaning that Fer-1 can block both pathways for oxPE formation. ${ }^{11}$ Acyl-coenzyme A (CoA) synthetase long-chain family member 4 (ACSL4) confers sensitivity to ferroptosis by selectively enriching membranes with PUFA, which are needed for ferroptosis execution. ${ }^{12}$ Also, it was recently discovered that peroxisomes can contribute to ferroptosis by synthesizing ether-linked PLs (so called plasmalogen). ${ }^{13} 14$ The plasmalogen from the peroxisome can be peroxidized as well, leading to ferroptosis. However, cells can downregulate the formation of plasmalogen, especially during cell state transitions, leading to ferroptosis resistance; this has been observed in carcinoma cells. ${ }^{15}$ These different pathways make ferroptosis a potential target for killing cancer cells and could be used in anticancer treatment.

\section{Morphological changes during ferroptosis}

Morphologically, ferroptosis is different from other cell death modalities, and especially from apoptosis. Ferroptosis is characterized mainly by mitochondrial aberrations. Shrunken mitochondria, reduced cristae, and rupture of the outer mitochondrial membrane are the most common manifestations. ${ }^{16}$ It should also be noted that the density of the mitochondrial membrane increases, ${ }^{1}$ and neither chromatin condensation nor blebbing occur. On the other hand, the main morphological changes in apoptosis are chromatin condensation and blebbing, together with cell shrinkage and nuclear fragmentation. ${ }^{17-19}$ Ferroptosis is also morphologically 
Table 1 Ferroptosis-inducing treatments as anti-cancer therapy

\begin{tabular}{|c|c|c|c|}
\hline Therapy & (Pre)clinical model & Target/antitumor mechanism of action & Ref. \\
\hline \multicolumn{4}{|l|}{ Liver cancer/HCC } \\
\hline Sorafenib+retinoblastoma KD & $\begin{array}{l}\text { BALB/c nude mice xenograft model } \\
\text { by subcutaneous injection of Huh7 } \\
\text { cells }\end{array}$ & $\begin{array}{l}\text { Sorafenib causes ROS production and lipid peroxidation. Sorafenib is } \\
\text { more cytotoxic in cells with reduced retinoblastoma protein levels. }\end{array}$ & 123 \\
\hline Artesunate + sorafenib & $\begin{array}{l}\text { BALB/c nude mice xenograft model } \\
\text { by subcutaneous injection of Huh7 } \\
\text { cells }\end{array}$ & $\begin{array}{l}\text { Artesunate and low-dose sorafenib have a synergistic effect in } \\
\text { inducing HCC ferroptosis in vitro and in vivo. Combined treatment } \\
\text { induced oxidative stress. }\end{array}$ & 124 \\
\hline Solasonine & $\begin{array}{l}\text { BALB/c nude mice xenograft model } \\
\text { by subcutaneous injection of HepG2 } \\
\text { cells }\end{array}$ & $\begin{array}{l}\text { Solasonine significantly suppresses expression of GPX4 and GSS } \\
\text { resulting in a defective GSH redox system, leading to ferroptosis. } \\
\text { Solasonine also inhibits cell migration. }\end{array}$ & 126 \\
\hline RSL3 +sorafenib & $\begin{array}{l}\text { GSTZ1-/- mice model, liver cancer } \\
\text { induced by intraperitoneal injection } \\
\text { of DEN followed by } \mathrm{CCl}_{4}\end{array}$ & $\begin{array}{l}\text { Sorafenib combined with RSL3 synergistically overcome resistance } \\
\text { to sorafenib acquired by GSTZ1 depletion. GSTZ1 inhibits the NRF2 } \\
\text { pathway. }\end{array}$ & 127 \\
\hline $\begin{array}{l}\text { Sorafenib+QSOX1 } \\
\text { overexpression }\end{array}$ & $\begin{array}{l}\text { BALB/c nude mice xenograft model } \\
\text { by subcutaneous transplantation } \\
\text { of MHCC } 97 \mathrm{H} \text { cells overexpressing } \\
\text { QSOX1 } \\
\text { HCC patient tissue samples from } \\
\text { liver resections }\end{array}$ & $\begin{array}{l}\text { QSOX1 promotes sorafenib-induced ferroptosis by suppressing NRF2 } \\
\text { expression. }\end{array}$ & 129 \\
\hline
\end{tabular}

Gastric carcinoma

ACP

Erastin $+\mathrm{CDO} 1$ overexpression Athymic nude mice xenograft model by subcutaneous injection of BGC823 cells

\section{HNC}

Artesunate +trigonelline Athymic BALB/c nude mice xenograft model by s.c. injection of HN9 cells

Sulfasalazine+cisplatin Athymic male BALB/c nude mice ${ }^{*}$ Cisplatin is FDA approved for xenograft model by subcutaneous treatment of NSCLC

$\mathrm{PGZ}+$ sulfasalazine injection of HN9 cells

Athymic BALB/c male nude mice xenograft model by subcutaneous injection of $\mathrm{HN} 10$ cells

Glutaredoxin 5

$\mathrm{KD}+$ sulfasalazine

Athymic BALB/c male nude mice xenograft model by subcutaneous injection of HN4R cells

MPC1 KD +sulfasalazine Athymic BALB/c nude mice xenograft model by subcutaneous injection of HN4 cells
ACP significantly inhibited proliferation and migration of gastric carcinoma cells in vitro, increased the accumulation of ROS, and suppressed xenograft growth and metastasis. ACPs inhibited the expression of GPX4 and System Xc-.

CDO1 mediates erastin-induced ferroptosis by converting cysteine in ${ }^{131}$ taurine, thereby decreasing GSH levels.

Artesunate can selectively induce ferroptosis in HNC cells. NRF2

silencing by trigonelline treatment increased artesunate sensitivity.

Sulfasalazine inhibits the system Xc- significantly, which sensitized 133 resistant $\mathrm{HNC}$ cells to cisplatin, and overcomes cisplatin resistance of HNC cells by inducing ferroptosis.

PGZ treatment reversed resistance to SAS-induced ferroptosis, by inhibiting NAF-1 leading to increased levels of mitochondrial ferrous iron and lipid ROS.

KD of GLRX5 increases the labile iron pool and sensitizes HNC cells 135 to SAS-induced ferroptosis.

Drug-tolerant persister cancer cells acquire mesenchymal traits, rendering them vulnerable to ferroptosis induction in vitro and in vivo. Regulation of a KDM5A-MPC1 axis (MPC1 KD) in cancer cells increased vulnerability to ferroptosis in vitro and in vivo by retaining mesenchymal traits and glutaminolysis.

\section{Lung cancer}

Cisplatin + RSL3

Athymic BALB/c nude mice

Inhibition of GPX4 by RSL3 enhanced the anticancer effect of xenograft model by direct injection of cisplatin in vitro and in vivo. Synergistic effect is induced by the A549 cells into the axilla cisplatin-regulated ferritinophagy.

Erianin

BALB/c nude mice xenograft model by subcutaneous injection of $\mathrm{H} 460$ cells
Erianin induced G2/M-phase arrest, inhibited cancer cell migration, and induced ferroptosis both in vitro and in vivo, accompanied by ROS accumulation, lipid peroxidation, and GSH depletion. 
Table 1 Continued

\begin{tabular}{lll}
\hline Therapy & (Pre)clinical model & Target/antitumor mechanism of action \\
\hline USP35 KD +erastin/RSL3 & BALB/c nude mice xenograft model & USP35 directly interacted with ferroportin and functioned as a \\
& $\begin{array}{l}\text { by subcutaneous injection of H460, } \\
\text { H1299 or H1650 cells }\end{array}$ & $\begin{array}{l}\text { deubiquitinase to maintain its protein stability. USP35 KD increased } \\
\text { intracellular LIP levels and promoted ferroptosis via decreasing }\end{array}$ \\
& $\begin{array}{ll}\text { Human lung adenocarcinoma tissues } \\
\text { and squamous cell cancer tissues }\end{array}$ & $\begin{array}{l}\text { ferroportin-mediated iron export, accompanied by the decrease of } \\
\text { lung cancer cell growth, colony formation, tumor progression in vitro } \\
\text { and in vivo. USP35 overexpression reduced erastin/RSL3-triggered }\end{array}$ \\
& $\begin{array}{l}\text { iron disturbance and ferroptosis, thereby facilitating lung cancer cell } \\
\text { growth and tumor progression. }\end{array}$
\end{tabular}

\section{NSCLC}

Acetaminophen (APAP) +erastin

Athymic BALB/c nude mice xenograft model by subcutaneous injection of A549 cells

Erastin +ectopic expression of Athymic BALB/c nude mice MT1DP

xenograft model, subcutaneous injection of A549 cells by intraperitoneal injection of erastin
APAP sensitized erastin-induced ferroptosis by regulating the Nrf2/ HO-1 signaling pathway, the cotreatment inhibited NSCLC cell viability and promoted ferroptosis and apoptosis, accompanied with attenuation of GSH and ectopic increases in lipid peroxides. The combination of erastin and APAP had favorable therapeutic effects on xenograft lung cancer.

Ectopic expression of MT1DP sensitized NSCLC cells to erastininduced ferroptosis through downregulation of NRF2. The cotreatment upregulated MDA and ROS levels, increased intracellular ferrous iron concentration and reduced GSH levels in cancer cells. In vivo analysis confirmed favorable therapeutic effect of erastin +MT1DP ectopic expression on lung cancer xenograft.

\section{Colorectal cancer}

Talaroconvolutin A (TalaA)

BALB/c nude mice xenograft model by s.c. injection of HCT116 cells, intraperitoneal injection of TalaA

TalaA kills colorectal cancer cells in a dose-dependent and timedependent manner by increasing ROS levels, downregulating the expression of SLC7A11, and upregulating ALOXE3, resulting in ferroptosis induction. Moreover, TalaA effectively suppressed the growth of xenografted colorectal cancer cells in vivo without obvious liver and kidney toxicities.

BALB/c nude mice xenograft model
by subcutaneous injection of DLD-1
cells

\section{Glioblastoma multiforme}

Temozolomide+quinolone NOD-SCID mice xenograft model by intracranial injection of GSC\#1
IMCA regulates the activity of the AMPK/mTOR/p70S6k signaling pathway. IMCA induced ferroptosis in colorectal cancer cells by downregulating the expression of SLC7A11, decreasing the contents of cysteine and GSH, and ROS accumulation. IMCA inhibited tumor growth in vivo with negligible organ toxicity.
In vitro, inhibition of autophagy with quinolone increases

temozolomide susceptibility. However, in vivo no significant increase
BALB/c nude mice xenograft model by subcutaneous injection of U251 cells

Dihydroartemisinin + epigallocatechine gallate

Athymic BALB/c nude mice xenograft model by subcutaneous injection of U251 or U373 cells

Pseudolaric acid B

Athymic BALB/c nude mice xenograft model by subcutaneous injection of $\mathrm{C} 6$ cells in anti-tumor effect was observed.

Amentoflavone triggered ferroptosis in an autophagy-dependent manner by increasing intracellular levels of iron, MDA and lipid ROS.

Dihydroartemisinin induced ferroptosis accompanied with ROS 146 generation and lipid peroxidation while simultaneously activating a negative feedback pathway of ferroptosis by increasing the expression of HSPA5, leading to increased expression and activity of GPX4. Epigallocatechine gallate inhibits HSPA5 and increased DHA sensitivity of glioma cells by increasing ferroptosis.

Pseudolaric acid B improved intracellular iron by upregulation of the transferrin receptor, which activated Nox4, resulting in overproduction of $\mathrm{H}_{2} \mathrm{O}_{2}$ and lipid peroxides. Moreover, pseudolaric acid $\mathrm{B}$ depleted intracellular GSH via p53-mediated xCT pathway. The in vivo data confirmed that the inhibitory effect of PAB on the growth of glioma cells was associated with increased intracellular iron and lipid peroxidation.

\section{Lymphoma}

Imidazole ketone erastin NCG mice xenograft model by subcutaneous injection of SUDHL-6 cells
IKE induced ferroptosis by inhibiting system Xc-, leading to GSH depletion and lipid peroxidation in vitro and in vivo. PEG-PLGA nanoparticles used to aid in IKE delivery had reduced toxicity in the mouse xenograft model.

(1)


Table 1 Continued

\begin{tabular}{|c|c|c|c|}
\hline Therapy & (Pre)clinical model & Target/antitumor mechanism of action & Ref. \\
\hline $\begin{array}{l}\text { Cisplatin + MAP30 protein } \\
\text { from Momordica charantia }\end{array}$ & $\begin{array}{l}\text { BALB/cAnN-nu nude mice xenograft } \\
\text { model by intraperitoneal injection of } \\
\text { ES2 cells }\end{array}$ & $\begin{array}{l}\text { MAP30, a natural AMPK activator, induced cell cycle arrest in the } \\
\text { S-phase, modulated cell metabolism, adipogenesis, and lipid droplet } \\
\text { formation in tumor development and progression, and induced an } \\
\text { increase in intracellular } \mathrm{Ca}^{2+} \text { concentration, which triggered ROS- } \\
\text { mediated cancer cell death via apoptosis and ferroptosis. MAP30 } \\
\text { showed a synergistic effect on cisplatin-induced cell cytotoxicity, and } \\
\text { coinjection resulted in a remarkable reduction of tumor dissemination } \\
\text { and tumor growth in the ovarian cancer ascites mouse model. }\end{array}$ & 149 \\
\hline Erastin +A939572 & $\begin{array}{l}\text { NOD/NSG mice xenograft model by } \\
\text { intraperitoneal injection of FT-t cells }\end{array}$ & $\begin{array}{l}\text { SCD1 alters lipid membrane composition and modulates ferroptosis. } \\
\text { Inhibition of SCD1 by A939572 modulates lipid metabolism and } \\
\text { enhances the antitumor effect of ferroptosis inducers in ovarian } \\
\text { cancer both in vitro and in vivo. }\end{array}$ & 150 \\
\hline Sulfasalazine +Olaparib & $\begin{array}{l}\text { BALB/c nude mice xenograft model } \\
\text { by subcutaneous injection of A2780 } \\
\text { or HEY cells }\end{array}$ & $\begin{array}{l}\text { PARP inhibition by olaparib promoted ferroptosis by repressing } \\
\text { SLC7A11, accompanied with decreased GSH biosynthesis and lipid } \\
\text { peroxidation, in ovarian cancer. Cotreatment with sulfasalazine in vivo } \\
\text { reduced tumor growth, prolonged mice survival and sensitized tumors } \\
\text { to olaparib. }\end{array}$ & 151 \\
\hline
\end{tabular}

Breast cancer/HER2+ breast cancer

$\begin{array}{lll}\text { Neratinib } & \begin{array}{l}\text { BALB/c mice model by injection of } \\ \text { TBCP-1 cells into the left cardiac } \\ \text { ventricle }\end{array} & \begin{array}{l}\text { Neratinib promoted ferroptosis in vitro. Metastasis assays in vivo } \\ \text { demonstrated that neratinib inhibited tumor growth and metastasis, } \\ \text { and prolonged survival. }\end{array} \\ \text { Melanoma } & \begin{array}{l}\text { Immunodeficient C57BL/6 nude mice } \\ \text { xenograft model by subcutaneous } \\ \text { injection of A375 or G-361 cells }\end{array} & \begin{array}{l}\text { miR-137 negatively regulates ferroptosis by directly targeting SLC1A5 } \\ \text { in melanoma cells, resulting in decreased glutamine uptake and MDA } \\ \text { accumulation. KD of miR-137 increased the antitumor activity of } \\ \text { Erastin by enhancing ferroptosis both in vitro and in vivo. }\end{array}\end{array}$

\section{Fibrosarcoma}

RSL3

Immunocompetent C57BL/6 J mice prophylactic tumor vaccination model by subcutaneous injection of ferroptotically dying MCA205 cells
RSL3 induced ferroptotic cell death in murine fibrosarcoma cells by inhibition of GPX4. Early ferroptotic cells were immunogenic both in vitro and in vivo.

ACP, Actinidia chinensis (Planch.); ALOXE3, arachidonate lipoxygenase 3; AMPK, AMP-activated protein kinase; APAP, acetaminophen; ATRA, all-trans retinoic acid; CDO1, cysteine dioxygenase 1; DEN/CCI4, diethylnitrosamine/carbon tetrachloride; FTH1, ferritin heavy chain 1; GLRX5, glutaredoxin 5; GSTZ1, glutathione S-transferase zeta 1; HCC, hepatocellular carcinoma; HO1, heme oxygenase-1; HSPA5, heat shock protein family A (Hsp70) member 5; IKE, imidazole ketone erastin; IMCA, 2-imino-6-methoxy-2H-chromene-3-carbothioamide; KD, knockdown; MDA, malondialdehyde; MPC1, mitochondrial pyruvate carrier 1; MT1DP, metallothionein 1D pseudogene; MT-1G, metallothionein-1G; NQO1, quinone oxidoreductase-1; NSCLC, non-small cell lung cancer; PEG-PLGA, polyethylene glycol-poly(lactic-co-glycolic acid); PGZ, pioglitazone; PPG, propargylglycine; QSOX1, Quiescin sulfhydryl oxidase 1; SAS, sulfasalazine; TalaA, talaroconvolutin A; USP35, ubiquitin-specific protease 35

different from necroptosis, which is characterized by swelling of the cells and intracellular organelles, and membrane rupture. ${ }^{182021}$

It was recently shown in human trophoblast cells (primary PHT cells and the BeWo human trophoblast cell line) that macroblebbing of the membrane occurred during ferroptosis. ${ }^{22}$ This macro-blebbing was caused by the increased membrane flexibility due to the presence of hydroxyperoxidated $\mathrm{PE}$, as shown by analyzing the elasticity of a simulated double lipid layer membrane. This blebbing was also confirmed by Van der Meeren et al by using atomic force microscopy ${ }^{18}$ In that study, membrane protrusions were observed after induction of ferroptosis in murine fibrosarcoma L929 cells and MCA205 cells, but this was accompanied by a decrease in overall elasticity of the cells. The more flexible oxPE might be responsible for bleb formation, but the elasticity of the cells still decreases during ferroptosis, presumably because the blebs containing oxPEs are released, causing overall decrease of elasticity. Of course, ferroptosis is also associated with cell rounding and plasma membrane permeabilization at the end of the cell death process. ${ }^{23}$ However, in necrosis induced by bacterial poreforming toxin, membrane permeabilization is an active process mediated by ninjurin-1. In ferroptosis, pores are formed by an unknown mechanism (thought to be lipid peroxidation-dependent) leading to osmotic rupture of the membrane. ${ }^{24-26}$ Interestingly, ferroptosis could propagate to neighboring cells independently of cell rupture by $\mathrm{Ca}^{2+}$ fluxes through the ferroptotic pores. ${ }^{26}{ }^{27}$ All these features make ferroptosis morphologically distinct from both apoptosis and necroptosis. This distinction might be used to morphologically distinguish ferroptosis from other regulated cell death modalities by using different microscopic methods, including those based on artificial intelligence. $^{28}$

\section{HALLMARKS OF IMMUNOGENIC CELL DEATH (ICD) Damage-associated molecular patterns}

Danger-associated molecular patterns (DAMPs) are endogenous intracellular molecules that in normal 

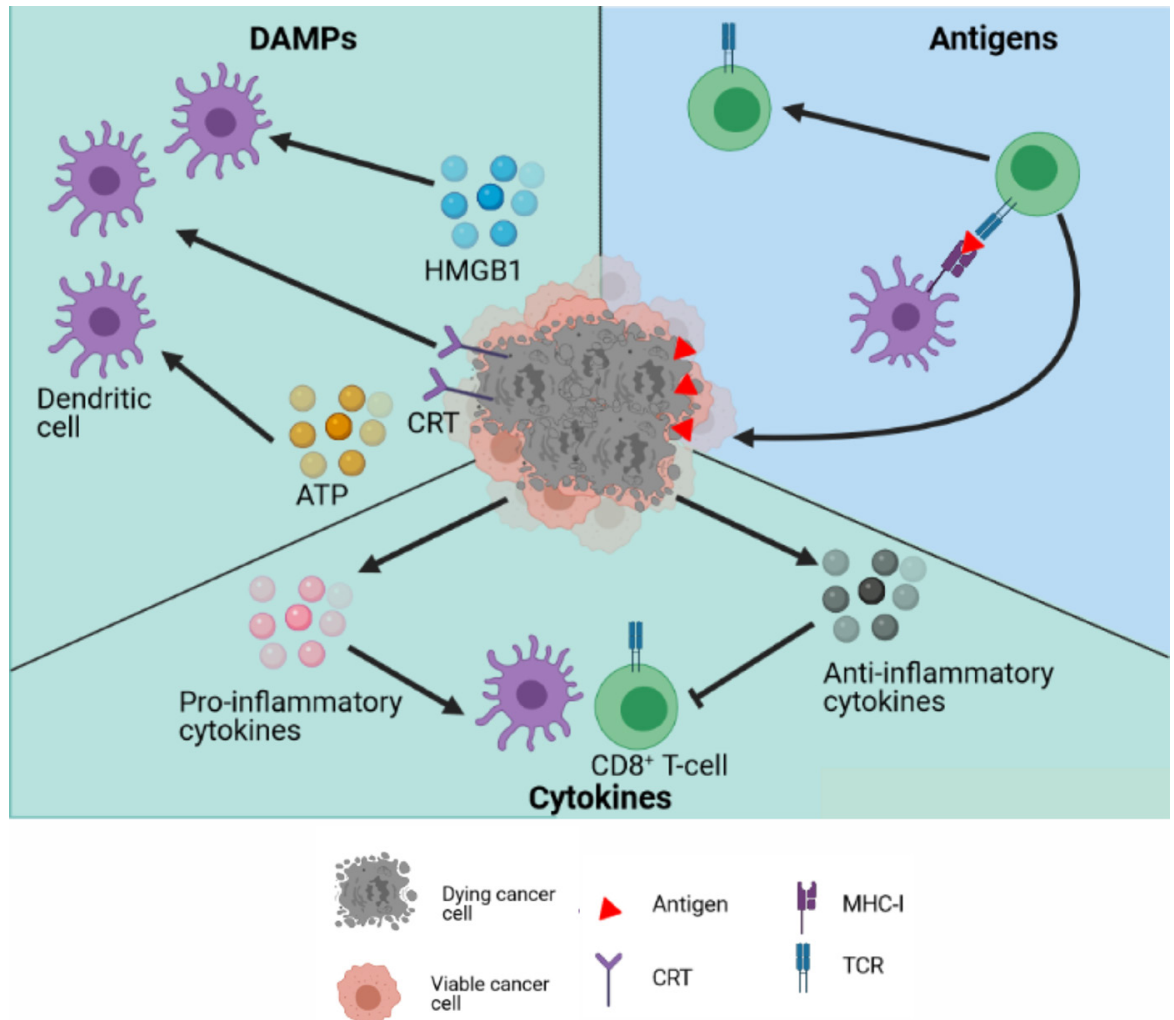

Figure 2 Hallmarks of ICD. The immunogenicity of cancer cell death is dependent on two main elements: adjuvanticity (green) and antigenicity (blue). HMGB1 binds to TLR4 on DCs, causing their activation and maturation. ${ }^{39} \mathrm{CRT}$ is expressed on the surface of dying (apoptotic) cells and will bind to LRP1 on DCs. ${ }^{40}$ ATP is either actively secreted or passively released by dying cancer cells in a specific spatiotemporal pattern and will bind to $\mathrm{P}_{2} \mathrm{X}_{7}$ receptors on DCs, causing their activation and maturation and leading to inflammasome activation. ${ }^{41}$ Proinflammatory cytokines act as adjuvants and can be seen as iDAMPs activating the immune system cells. ${ }^{50}$ Anti-inflammatory cytokines cause inhibition of immune cells and are considered protumorigenic. Antigens are presented by DCs on MHC-I molecules to T cells in a process called cross-presentation. ${ }^{58}$ After antigen recognition, cytotoxic $\mathrm{CD}^{+} \mathrm{T}$ cells are formed and migrate to the tumor, where they eliminate antigen-expressing cancer cells. ${ }^{57}$ Memory $T$ cells may be formed, establishing long-lasting antitumor immune responses and reducing the chance of tumor reoccurrence. CRT, calreticulin; DAMP, danger-associated molecular pattern; DC, dendritic cell; HMGB1, high-mobility group 1; ICD, immunogenic cell death; iDAMP, inducible danger-associated molecular pattern; LRP1, lipoprotein receptor-related protein 1 ; TCR, T-cell receptor.

conditions contribute to different physiological processes. They are released (or exposed on the outer surface of the plasma membrane) when a cell is damaged or dying. ${ }^{29}$ Once released, DAMPs acquire immunostimulatory properties and bind to pattern-recognition receptors, such as Toll-like receptors (TLRs, eg, TLR4) and purinergic receptors $\left(\mathrm{P}_{2} \mathrm{X}_{7}\right)$ to promote inflammation and activation of the innate immune system. DAMPs originate from the cytosol and mitochondria, and are extracellular matrix (ECM)-derived. In contrast to pathogen-associated molecular patterns, DAMPs are not associated with pathogens. ${ }^{30}$ The most well-known DAMPs, which play an important role as adjuvants in ICD, are ATP, high-mobility group 1 (HMGB1) and calreticulin (CRT) (figure 2). These molecules bind to $\mathrm{P}_{2} \mathrm{X}_{7}$ receptors, TLR4 or low-density lipoprotein receptor-related protein 1, respectively. By activating the immune system, DAMPs can enhance antitumor immunity during and after cancer treatment. ${ }^{31} 32$

DAMPs are an important component in ICD by providing adjuvanticity to dying cells. ${ }^{33}$ It has been established that DAMPs are emitted at a precise moment and that the release of all the DAMPs in a specific sequence is required for effective activation of the immune system. Apoptosis was previously thought to be tolerogenic, ${ }^{34}$ but in certain cases, and more specifically in apoptotic cancer cells, it could activate the immune system. ${ }^{35-38}$ This immunogenic apoptosis was associated with the release of HMGB1 and exposure of CRT, thus causing immune cell activation. ${ }^{39-41}$ HMGB1 and ATP release have also been observed in necroptosis. ${ }^{4243}$ This indicates that both apoptosis and necroptosis show hallmarks of ICD and could be exploited in cancer therapy. However, cancer cells are known to be resistant to those cell death types, which diminishes their therapeutic potential. ${ }^{44}$ Therefore, there is a need for triggering other cell death modalities.

\section{Cytokines released during ICD}

DAMPs are not the only factors released during cell death that have adjuvant properties. Cytokines are a diverse group of molecules that can be either proinflammatory or 
anti-inflammatory. ${ }^{45}$ Proinflammatory cytokines can act as adjuvants too during ICD, whereas anti-inflammatory cytokines dampen the immune response directed at the dying cells (figure 2). Interestingly, cytokines are more effective than DAMPs as they function at lower concentrations in the range of picogram per millilitre. ${ }^{46}$ For example, in one study, the DAMP heat shock protein 70 (HSP70) was found to be expressed at the nanogram scale, whereas the cytokines (mainly interleukins) were in the picogram scale. ${ }^{46}$ However, a clear distinction between proinflammatory and anti-inflammatory cytokines cannot be made, as many of them share common signaling pathways and the eventual outcome depends on the cell type, the presence of other cytokines, and the tumor microenvironment. In this regard, Abiko et al showed that in tumors resected from patients with ovarian cancer, the tumor-promoting factor prodeath ligand 1 was expressed more strongly on tumor cells due to increased secretion of interferon gamma (IFN- $\gamma$ ) by $\mathrm{CD} 8{ }^{+} \mathrm{T}$ cells. ${ }^{47}$ However, IFN- $\gamma$ can also have a tumor-suppressive effect due to its upregulation of antigen processing and presentation. ${ }^{48}$ This might be explained by the more complex microenvironment in the tumors, where different cell types interact with each other and the eventual effect on the immune system depends on the overall balance between proinflammatory and anti-inflammatory factors. ${ }^{49}$

It should be noted that cytokine secretion is induced during cell death (eg, necroptosis), and so these cytokines can be seen as inducible danger-associated molecular patterns (iDAMPs, as proposed by Yatim et $a \tilde{l}^{\tilde{0}}$ ). Together with the DAMPs already present inside cells (such as ATP and HMGB1), iDAMPs are released during cell death, after which they interact with immune cells to induce activation of the immune system. Thus, both DAMPs and iDAMPs are required for efficient immune activation. While DAMPs are generally viewed as one of the main determinants of immunogenicity, the presence of iDAMPs suggests that immunogenicity not only depends on the release of DAMPs but also requires stressinduced transcription of proinflammatory cytokines. ${ }^{51}$ Moreover, cytokines (eg, type I interferons) might act in an autocrine fashion on cancer cells to increase their immunogenicity. ${ }^{52}$ Therefore, analysis of the cytokine secretion pattern is important in assessing the immunogenicity of cell death.

During and even after cell death, cytokines can have a significant effect on its immunogenicity. It has been observed that even when the cell membrane has been permeabilized in necroptotic cells, the corpses still release cytokines, such as interleukin (IL)-6, CXCL1, CXCL2 and CCL2due to RIPK3 activation. ${ }^{53}$ This continued cytokine secretion upregulates phagocytosis by bone marrow-derived macrophages and enhances the immune response by promoting cross-presentation (presentation of antigen in the context of major histocompatibility complex (MHC)-I molecules). Thus, the cytokines act as adjuvants during ICD. However, previous studies have shown that when macrophages take up necroptotic L929 fibrosarcoma cells induced by tumor necrosis factor-alpha $(\mathrm{TNF}-\alpha)$, no proinflammatory cytokines are produced and an effective immune response is not generated. ${ }^{19} 54$ This might have been due to the use of different inducers. Orozco $e$ t $a \bar{l}^{\tilde{3}}$ used a cell line with a constitutively active RIPK3 to trigger necroptosis, whereas Brouckaert et al induced necroptosis with TNF- $\alpha .{ }^{54}$ The two methods might induce different expression patterns of cytokines and thereby lead to different immune responses. The interaction of different stress-induced signaling pathways will play an important role in the eventual immunogenicity of dying cells.

Different cell death modalities eventually lead to different immunogenicity outcomes. For efficient ICD, all the previously mentioned parameters, such as DAMPs and iDAMPs (responsible for adjuvanticity) and antigens (for antigenicity, see next section), need to be present in a specific spatiotemporal pattern. For example, Zhang et al showed in CT26 cells that ATP and HMBG1 release from apoptotic cells peaked at 6 hours and from necroptotic cells at 8 hours. ${ }^{55}$ Also, CRT expression at the membrane was observed after an hour of necroptosis induction. In strong contrast, in apoptotic MCF7 cells, ATP and HMGB1 release peaked only after 12 and 48 hours, respectively, ${ }^{56}$ and membrane CRT expression was observed only after 10 hours. Despite these differences, both cell death modalities have been shown to be immunogenic in nature. This means that different cell death modalities may differ in their modulation of DAMP exposure, leading to activation of the immune system. This highlights the importance of the cell death type in ICD. The hallmarks of ICD should always be studied in terms of time kinetics and pattern of DAMPs, iDAMPs and antigen expression in order to properly correlate these variables with the responses to ICD.

\section{Modulation of antigenicity during ICD}

Another major determinant of immunogenic cancer cell death is antigenicity. ${ }^{33}$ It is known that efficient induction of antitumor immunity requires exposure of the relevant antigens. Antigens can be derived from intracellular proteins that become degraded to peptides by proteasomes. ${ }^{57}$ The peptides are transported to the endoplasmic reticulum, where they bind to newly produced MHC-I molecules. The MHC-I molecules, with the peptides bound in their groove, are transported to the plasma membrane, where they can be recognized by $\mathrm{T}$ cells. Normally, antigens bound to MHC-I are self-antigens that do not activate $\mathrm{T}$ cells because they have been selected for self-recognition during their development. Therefore, normal cells do not elicit an immune response, except in autoimmune diseases. However, since tumors may contain a large number of mutations, the mutated antigens presented by tumor cells are recognized as foreign.

The main cells capable of antigen presentation are dendritic cells (DCs), ${ }^{58}$ which can take up tumor antigens from dying cancer cells, and in the presence of DAMPs and iDAMPs become mature and activated. $\mathrm{CD} 4^{+}$helper 
$\mathrm{T}$ cells are required for efficient activation via the costimulatory molecules CD40/CD40L (figure 2). Activated DCs migrate to the lymph nodes and prime naive cytotoxic $\mathrm{CD}^{+} \mathrm{T}$ cells. Antigen-specific cytotoxic $\mathrm{T}$ cells might be formed, which can migrate back to the tumor, recognize the tumor cells presenting mutated antigens on MHC-I molecules, and kill them, thus leading to eradication of the tumor. Cross-presentation is the corner stone of cancer immunotherapy because it can lead to long-lasting protection against tumors. Of course, tumor cells have developed mechanisms of resistance to cross presentation, such as downregulation of MHC-I expression. ${ }^{57}$

Antigens can be tumor-specific antigens (TSAs), tumorassociated antigens (TAAs) or neoepitopes. ${ }^{59} 60$ TSAs are specific only for tumors. However, many cancers have developed resistance mechanisms to eliminate TSA expression in order to remain hidden from immune surveillance, for example, by downregulating the expression of TSAs and thereby eliminating antigen presentation. ${ }^{60}$ TAAs can also be used for antigen presentation, but this type of antigen is not specific for tumor cells. They are mainly proteins that are normally expressed only during embryogenesis or in immune privileged areas such as the brain or gonads. ${ }^{5961}$ Therefore, TAAs are less suitable for ICD-based therapies as the immune cells do not have sufficient specificity for the cancer. On the other hand, neoepitopes arise from mutations in cancer cells and are therefore specific for the tumor. ${ }^{61}$ This makes neoepitopes suitable for use in therapy because a specific immune response can be mounted against the cancer. However, the process of identifying neoepitopes remains very technically challenging. Also, in the tumor, there is a natural selection process of cancer cells that express limited antigens, and this reduces the efficacy of activating the immune system. ${ }^{62}$

The cell death type may also modulate antigenicity during ICD, leading to different therapeutic outcomes. Many cell lines (eg, colon carcinoma CT26 cells) used in the field of ICD already express non-endogenous antigens (eg, virally derived peptides), and thus, they will likely be recognized by the immune system as TSAs. ${ }^{63}$ In this context, it has been shown that both apoptotic and necroptotic CT26 colorectal cancer cells, which express the virally derived peptide AH1 (an immunodominant $\mathrm{Ag}$ ), can activate the immune system of mice and protect against the tumor. ${ }^{64}$ However, when a knock-out model for AH1 was used, only necroptotic CT26 cells were still immunogenic, whereas the apoptotic CT26 cells could not activate the immune system. The authors showed that only necroptotic CT26 cells could stimulate the formation of T cells specific for CT26 neoantigens, which means that necroptosis is a form of ICD with very strong immunogenic potential. While apoptosis seemed to be immunogenic, this was only caused because of the AH1 antigen, because when this antigen was removed, antigenicity was insufficient for efficient immune activation. Importantly, the type of cell death can also affect the antigenicity of dying cancer cells and thereby modulate their immunogenicity. The models should be selected critically because non-endogenous immunodominant antigens might mask the intrinsic antigenicity of different cell death modalities.

In conclusion, ICD is a complex phenomenon that requires at least three factors to be present in a specific spatiotemporal pattern (figure 2). To study ICD, both adjuvanticity and antigenicity need to be investigated as both are at least equally important features necessary for efficient activation of the immune system against the tumor (figure 2). Either of them alone will not lead to ICD but to an immunologically silent form of cell death. As ICD leads to activation of immune cells, and possibly to establishment of immunological memory, this might be an effective alternative for current immunotherapies or as part of a combined treatment modality. Current anticancer therapies mainly trigger apoptosis, but since resistance against apoptosis is a frequent occurrence in cancer, ${ }^{44}$ combined with the fact that antigenicity might be hindered during apoptosis, ${ }^{64}$ this might not be the ideal choice. Therefore, it is important to investigate whether other cell death modalities, such as necroptosis and ferroptosis, are immunogenic and to exploit them in immunotherapy in more clinically relevant models.

\section{FERROPTOSIS: A NEW CELL DEATH TYPE IN THE ICD FAMILY}

Initially, it was discovered that ferroptosis can be selectively triggered in cancer cells expressing oncogenic mutants of rat sarcoma protein (RAS), and thus it might be an attractive alternative to apoptosis and necroptosis for overcoming cell death resistance and enhancing the efficacy of anticancer therapy. ${ }^{1}$ Moreover, it was recently shown that ferroptotic cancer cells are a rich source of DAMPs such as HMGB1 and ATP, which could function as adjuvants and induce activation and maturation of DCs (discussed in the next section). This means that ferroptotic cancer cells might be used in cell-based anticancer immunotherapy. ${ }^{656}$ It was also discovered that in electron microscopy, ferroptotic cells exhibit an electron lucent nucleus, indicating nuclear membrane damage leading to the release of HMGB1, an important DAMP. ${ }^{67}$ Interestingly, this nuclear membrane damage occurred before cytoplasmic membrane rupture.

\section{Ferroptosis and innate immunity}

Ferroptosis is associated with inflammation and innate immunity. $\mathrm{Li}$ and colleagues ${ }^{68}$ demonstrated that ferroptotic cell death occurs in the early phase of reperfusion during intestinal ischemia-reperfusion injury (IRI). ${ }^{68}$ In that study, the authors showed that the expression of ACSL4, a key enzyme regulating lipid composition, was upregulated under ischemic conditions and that it contributed to reperfusion-induced ferroptotic injury. Though a direct link between inflammation and ferroptosis was not studied in that work ${ }^{68}$ the study suggested that IRI-induced ferroptosis might lead to infiltration of immune cells in the injured intestine. In another study 
related to myocardial IRI, ${ }^{69}$ it was demonstrated that inhibition of cell death with Fer-1 in the heart model subjected to IRI leads to reduction of several oxidized lipids associated with ferroptosis. The authors discovered that ferroptotically dying cells trigger TLR4/Trif signaling in endothelial cells, which promotes recruitment of neutrophils to the injured heart. The relevance of ferroptosis to mediating innate immunity has also been demonstrated in acute renal tubular necrosis and IRI, ${ }^{70}$ where leukocyte infiltration in the model of cremaster muscle IRI was observed. Moreover, infiltration into the postischemic area after treatment with Fer-1 was less than in controls. ${ }^{70}$ The aforementioned studies demonstrate an emerging role of ferroptotic cell death in the innate inflammatory response during tissue injury and IRI. However, this needs further examination to identify the main players and executors of ferroptosis-induced inflammation during IRI.

\section{DAMPs in ferroptotic cancer cell death}

\section{High-mobility group 1}

Release of DAMPs during ferroptotic immunogenic cancer cell death is being actively studied and has been described for several DAMPs, such as HMGB1 ${ }^{65} 667172$ and $\mathrm{ATP}^{65}$ (figure 2). HMGB1 release from ferroptotic cells was detected for the first time by Wen et al in human fibrosarcoma HT1080, human pancreatic carcinoma cells PANC-1 and immortalized mouse embryonic fibroblasts (MEF) following treatment with two different classes of ferroptotic inducers: class I (erastin and sorafenib) and class II (RSL3 and FIN56) ${ }^{66}$ Of note, the release of HMGB1 from ferroptotic cells was significantly decreased when ATG5 and ATG7, genes regulating the autophagy pathways, had been knocked out, suggesting that the autophagy pathway is required for the release of HMGB1 in ferroptosis. Importantly, extracellular HMGB1 released by dying cancer cells is a potent inducer of innate and adaptive immunity and is one of the main players in mediating the adjuvanticity of ICD (figure 2). HMGB1 binds to AGER/RAGE ${ }^{73}$ and TLR4 ${ }^{74}$ on the surface of antigen-presenting cells, driving the immune response against cancer cells. Initially, it was shown that HMGB1 evoked immunogenicity of apoptotic cancer cells via the MYD88/TLR4 signaling pathway. ${ }^{39} 75$ However, Wen and colleagues showed that in ferroptotic MEF cells, HMGB1mediated inflammation requires signaling through AGER/RAGE but not TLR4. ${ }^{66}$ How dying cancer cells prioritize HMGB1-mediated inflammation and decide between AGER/RAGE or TLR4 signaling remains unknown and needs further investigation.

Recently, we described the release of HMGB1 from mouse ferroptotic fibrosarcoma MCA205 cells (figure 3) induced with RSL3 (figure 1) ${ }^{65}$ We distinguished between 'early' and 'late' ferroptotic cells based on Annexin V/ Sytox Blue staining. Early ferroptotic cells (1 hour after ferroptosis induction) had 2x higher single Annexin $\mathrm{V}^{+}$population and $11 \mathrm{x}$ lower Annexin $\mathrm{V}^{+}$Sytox Blue ${ }^{+}$ population than late ferroptotic cells (24hours after stimulation with RSL3). We found that HMGB1 release gradually increased during the progression of cancer cells towards late ferroptosis and was maximal 24 hours after RSL3 stimulation, when all cancer cells were stained double positive for Annexin V and Sytox Blue. Moreover, to better mimic the situation in situ, the levels of HMGB1 were measured by co-culturing early and late ferroptotic cells with bone-marrow derived dendritic cells (BMDCs). We found that the level of HMGB1 was maximal when the BMDCs were co-cultured with early ferroptotic cancer cells, indicating the immunogenicity of early but not late ferroptotic cancer cells (table 1). Thus, these two studies $^{6566}$ describe the role of HMGB1 mainly as DAMPs that can contribute to the immunogenic potential of ferroptotic cancer cells (figure 3), similar to their role in the immunogenicity of other regulated cancer cell death modalities such as apoptosis ${ }^{39}$ and necroptosis. ${ }^{43}$ All these data confirm that HMGB1 is a key DAMP in different ICD modalities involved in the activation of innate ${ }^{76}$ and adaptive immunity. ${ }^{39}$

Unexpectedly, another study discovered a role of HMGB1 not related to immunogenicity but to regulation of ferroptosis. ${ }^{72}$ Ye and colleagues generated knockdown of HMGB1 in the HL-60 human acute myeloid leukemia line cell expressing NRAS ${ }^{\mathrm{Q} 61 \mathrm{~L}}$ and analyzed the capacity of the cells to undergo ferroptosis. Interestingly, when HMGB1-deficient HL-60 cells were treated with erastin (inhibitor of the cystine-glutamate antiporter system $\mathrm{X}_{\mathrm{C}}^{-}$, figure 1), both ROS generation and cell death via an iron-mediated lysosomal pathway decreased. These data suggest that in addition to its function as a DAMP, ${ }^{6566}$ HMGB1 is also a positive regulator of ferroptotic cell death itself. One can speculate that human tumors deficient in HMGB1 (eg, human breast cancers) ${ }^{77}$ are not only deficient in induction of anti-tumor immunity because ferroptosis would not be immunogenic, ${ }^{65}$ but also that such tumors would be resistant to ferroptosis. Future studies will shed light on the dual role of HMGB1 in ferroptosis as a DAMP and as a regulator of ferroptosis (figure 3), and additional work is needed to validate the possible dual role of HMGB1 in ferroptosis in human cancers.

\section{ATP}

While HMGB1 release from ferroptotic cells, its kinetics and immunogenic potential are currently being investigated, knowledge about ATP release during ferroptotic cell death is deficient. The dynamic range of ATP release from ferroptotic cancer cells has been recently reported ${ }^{65}$ (figure 3): extracellular ATP concentrations were measured in fibrosarcoma MCA205 cells throughout the course of ferroptotic cell death. ATP release gradually increased from 1 hour after ferroptosis induction with RSL3 (early ferroptotic cells) until 6 hours after induction. Interestingly, 24 hours after induction (late ferroptotic cells), ATP could no longer be measured and was probably depleted. In the murine tumor prophylactic vaccination model, the authors used early ferroptotic cells and demonstrated that if $\mathrm{P}_{2} \mathrm{X}_{7}$ purinergic channels 


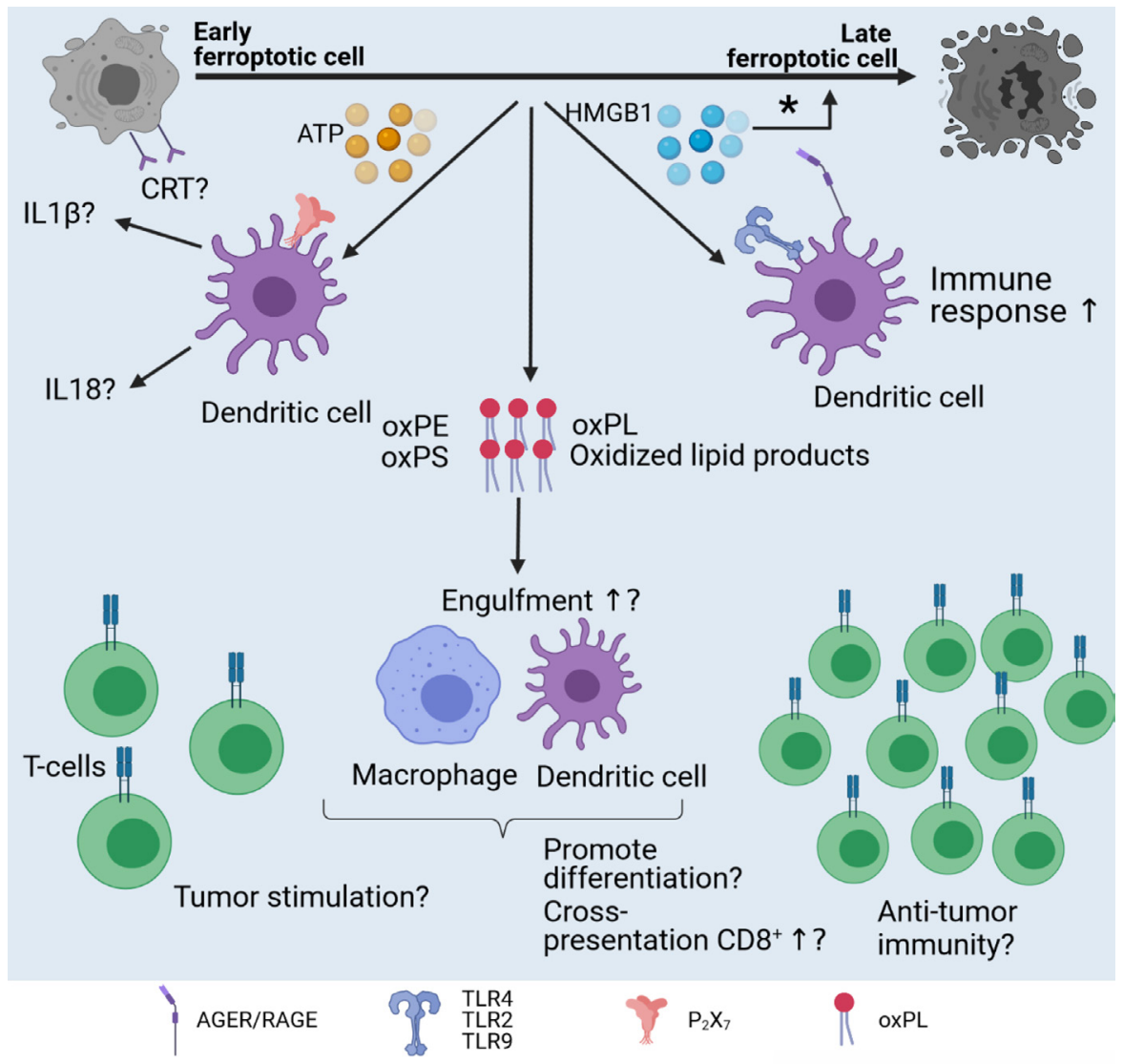

Figure 3 Immunogenic potential of ferroptosis. Several factors might influence the immunogenicity of ferroptosis. Early, but not late, ferroptotic cancer cells are immunogenic in vitro and in vivo, and HMGB1 and ATP are released by early ferroptotic cancer cells.$^{65}$ it is unknown if CRT is exposed on early ferroptotic cells, and this requires investigation. Notably, HMGB1 and ATP are not released by late ferroptotic cells. HMGB1 might act through the AGER/RAGE or TLR2/TLR4/TLR9 pathway and activate innate and adaptive immunity. Moreover, knockdown of the HMGB1 gene decreases erastin-induced ferroptosis, ${ }^{72}$ therefore pointing to the regulatory role of HMGB1 in ferroptosis $\left(^{*}\right)$. Another component potentially modifying the immunogenicity of ferroptotic cell death might hide behind oxPLs and other oxidized products generated during ferroptosis. OxPLs (eg, oxPE) can play a dual role in ferroptosis, inducing either tumor promotion or tumor suppression. However, the role of oxidized oxPLS in the immunogenicity of ferroptosis is not known. CRT, calreticulin; HMGB1, high-mobility group 1; IL, interleukin; oxPE, oxidized phosphatidylethanolamine; oxPL, oxidized phospholipid; oxPS, oxidized phosphatidylserine.

are blocked by the 2',3'-dialdehyde derivative of ATP (oxidized ATP), mice develop tumors at the challenge site more quickly, indicating that early ferroptotic cancer cells provide limited protection. ${ }^{65}$ This finding is in line with the role of ATP in the immunogenicity of apoptosis. When oxidized ATP was injected into mice, it reduced the immunogenicity of apoptosis in the tumor prophylactic vaccination model. Indeed, signaling through the $\mathrm{P}_{2} \mathrm{X}_{7}$ purinergic channels is required for the immunogenicity of apoptosis and activation of inflammasomes, leading to production of IL-1 $\beta$ and IL-18. ${ }^{78} 79$ Of note, when ATP fails to bind to the purinergic receptors due to their blockage or when $\mathrm{P}_{2} \mathrm{X}_{7}$ or $\mathrm{P}_{2} \mathrm{Y}_{2}$ is knocked out, recruitment of antigen-presenting cells and antitumor immune responses are abolished. ${ }^{67980}$ Additional studies are needed for a clear understanding of whether the ATP released from ferroptotic cancer cells will also lead to activation of inflammasome.

Also, a very recent article showed, in the model of murine prostate adenocarcinoma (TRAMP-C1) cells, that besides releasing ATP and HMGB1 during ferroptosislike cell death, the cells also exposed CRT. ${ }^{81}$ In that work, the Fenton reaction was induced using vesicles containing ascorbic acid and iron oxide nanocubes. CRT exposure significantly increased 6 hours post-treatment of TRAMP-C1 cells. The authors additionally showed that the treatment mediated anticancer immune responses in vivo. All these data together strongly indicate that at least three key DAMPs (HMGB1, ATP, and CRT) play essential roles in the immunogenicity of ferroptotic cancer cells and the induction of efficient antitumor immunity.

\section{Are oxPEs species required for the immunogenicity of ferroptosis?}

In addition to well-known DAMPs, such as CRT, HMGB1, ATP, and IFNs (figure 2), oxidized phospholipids (oxPLs) (figure 1) might also play an important modulatory role in the immunogenicity of ferroptotic cancer cells and in the subsequent immune responses. ${ }^{42}$ The execution of ferroptosis includes oxidation of different types of PLs in 
the plasma membrane. ${ }^{5} 128384$ During ferroptosis, enzymatic oxidation occurs, primarily of $\mathrm{PE}$, due to shortage of antioxidant protection (GPX4) and/or LOX-mediated PL peroxidation ${ }^{51283}$ (figure 1). There is no knowledge available yet about a direct influence of oxPL derived from ferroptotic cells and their role in modulating the immune system. However, in several recent studies, it has been shown that oxPL derived from or associated with other regulated cancer cell death modalities might affect the function of APCs and modulate the immune responses. In this regard, it has been reported that the oxidized version of externalized phosphatidylserine (PS) positively affects the engulfment of apoptotic cells by macrophages. ${ }^{85} 86$ Tyurin and colleagues ${ }^{85}$ compared the efficiency of the engulfment of apoptotic wild-type HL-60 cells (low in oxidized phosphatidylserine (oxPS)) and linoleic acid (LA)-enriched HL-60 cells (high in oxPS) by RAW264.7-derived and THP-1-derived macrophages. In apoptotic LA-HL-60 cells, increased oxPS led to enhanced phagocytosis by macrophages compared with control HL-60 cells. Not only oxPS but also oxPE, which is generated during ferroptosis (figure 1), can interfere with the clearance of apoptotic cells by professional phagocytes. ${ }^{87}$ Uderhardt and colleagues ${ }^{87}$ demonstrated that milk fat globule-EGF factor 8 (MFG-E8)-dependent uptake of apoptotic cells by inflammatory macrophages was limited in the presence of $\mathrm{PE}$ oxidation products. The authors showed that $\mathrm{PE}$ oxidation products, mediated by resident macrophages expressing 12/15-lipoxygenase (12/15LO), blocked phagocytosis of apoptotic cells. Interestingly, in the absence of $12 / 15$-LO, clearance of apoptotic cells by inflammatory monocytes was restored, leading to T-cell responses against antigens derived from the apoptotic cells. Considering these data and knowing that PE is one of the major PLs undergoing oxidation during ferroptosis (figure 1), it is conceivable that the engulfment of ferroptotic cancer cells by APC, such as DCs and macrophages, could be modulated by oxPE and its oxidation products (discussed in the next section). Although several studies have already demonstrated that ferroptotic cancer cells are efficiently engulfed by APCs, ${ }^{65} 88$ the role of oxPEs was not addressed. Thus, the effect of oxPEs generated during ferroptosis on phagocytosis and the immunogenicity of ferroptotic cancer cells are unknown, and additional studies can bring further insights into the role of oxPL in the immunogenicity of ferroptotic cancer cells (figure 3 ).

Importantly, it is not only the oxidation products derived from dying cancer cells that can affect the activity of antigen-presenting cells, because tumor-resident immune cells themselves can produce oxidized lipids, which affect other APCs and thus further modulate the anticancer immune responses. In this regard, it has been shown that cancer-associated DCs accumulated lipids in so-called lipid bodies, which weakened the function of DCs, and so they were not able to effectively stimulate antigen-specific T cells. ${ }^{89} 90$ This might have been mediated by the oxidative products in the lipid bodies, such as oxidatively truncated electrophilic lipids, which interact with the major stress-induced peptide chaperone HSP70, thereby preventing the presentation of peptides-MHC-I on the DC surface. ${ }^{89}$ Of note, it was recently demonstrated that polymorphonuclear myeloid-derived suppressor cells (PMN-MDSCs) interfere with cross-presentation of DCs, the process of interference being associated with lipid transfer. ${ }^{91}$ PMN-MDSC, a part of tumor microenvironment, produces a large amount of ROS, which generate various species of oxidized lipids, such as oxidized triacylglycerols or prostaglandin E2, which might affect DCs and impair the induction of specific antitumor $\mathrm{CD}^{+}$ T-cell responses. In another intriguing study, it has been reported that immunotherapy-activated $\mathrm{CD}^{+} \mathrm{T}$ cells promote tumor cell lipid peroxidation and sensitize tumors to ferroptosis through production of IFN- $\gamma .{ }^{92}$ In their study, Wang and colleagues demonstrated that IFN- $\gamma$ released from $\mathrm{CD} 8^{+} \mathrm{T}$-cells downregulates the expression of SLC3A2 and SLC7A11 (subunits of the glutamatecystine antiporter system $\mathrm{X}_{\mathrm{C}}^{-}$) and diminishes the uptake of cystine by tumor cells, and thus promotes cancer cell lipid peroxidation and ferroptosis. Altogether, these results show an important role of lipid oxidation products in shaping the response of the adaptive immune system to cancer. There is a lot of speculation about probable effects of ferroptotic cancer cells, which contain large numbers of various oxidized and peroxidized lipids and PLs, ${ }^{5128384}$ on DCs, CD8 ${ }^{+}$T cells and, overall, on the anticancer immune response. Therefore, future studies are needed to clarify whether ferroptosis-derived oxPL are responsible for modulating the immunogenicity of ferroptosis and to explain the non-immunogenicity of late ferroptotic cells ${ }^{65}$ (figure 3).

\section{FERROPTOSIS AND THE TUMOR MICROENVIRONMENT Effect of ferroptosis on macrophages}

As mentioned previously, ferroptosis affects immune cells in the tumor microenvironment by activating DCs. ${ }^{65}$ However, the tumor microenvironment is very complex and contains other cell types. ${ }^{93}$ Ferroptosis might also affect the other cells in the tumor microenvironment. It has been shown that macrophages can behave differently during ferroptosis induction. ${ }^{94} \mathrm{M} 1$ macrophages, which are considered proinflammatory, are very resistant to ferroptosis because of their high levels of inducible nitric oxide synthase. This is in contrast to the alternatively activated M2 macrophages, which are considered antiinflammatory and are more sensitive to ferroptosis. Secretion of nitric oxide $(\mathrm{NO} \bullet)$ inhibits the 15LOX-catalyzed peroxidation of PUFAs in M2 macrophages, thus blocking ferroptosis execution. Hence, ferroptosis can also kill the tumor-promoting M2 macrophages, removing their immunosuppressive effects while preserving the immunostimulatory role of M1 macrophages. However, M1 macrophages are not only more resistant to ferroptosis but can also confer this resistance to other cells ${ }^{94}$ and contribute to inflammation-driven carcinogenesis. It has 
been shown that mouse lung epithelial cells were more resistant to ferroptosis in the presence of M1 macrophages due to NO• secretion. ${ }^{94}$ It is unknown if they can also confer this resistance to cancer cells, which would reduce the therapeutic efficiency of ferroptosis. This is in stark contrast to the widely accepted notion that M1 macrophages possess antitumoral effects. Notably, ferroptotically dying cancer cells can also directly affect macrophages. An intriguing aspect of ferroptosis was recently presented in regard to polarization of tumor-associated macrophages. ${ }^{95}$ The authors showed that autophagydependent ferroptosis of cancer cells leads to procancer polarization of pancreatic tumor-associated macrophages. The process was shown to be regulated through the AGER/RAGE pathway and STAT3-dependent fatty acid oxidation. Ferroptotic pancreatic ductal adenocarcinoma cells release $\mathrm{KRAS}^{\mathrm{G} 12 \mathrm{D}}$, which is taken up by macrophages and causes them to switch to an M2-like protumor phenotype.$^{95}$ Whether this effect is characteristic of all types of cancer cells undergoing ferroptosis awaits thorough investigation. By modulating the switch between M1 and M2 macrophages, the immune system might be more efficiently activated and might generate strong and longlasting antitumor immunity.

\section{Lactate and acidification}

Lactate is an important metabolite abundant in the tumor microenvironment because cancer cells often switch to glycolysis. ${ }^{96}$ However, it was recently shown that lactate is not merely a waste product but can be used by the cancer cells to promote ferroptosis resistance. ${ }^{97}$ Lactate uptake causes an increase in ATP production, leading downstream to upregulation of stearoyl-CoA desaturase-1, which produces monounsaturated fatty acids. Lactate can also directly inhibit ACSL4, which, as mentioned earlier, can enrich PUFAs in the plasma membrane. This combined effect thus decreases the available PUFAs needed for ferroptosis and thereby inhibits cell death. In addition, lactate acidifies the tumor microenvironment, leading to increased iron storage and decreased iron availability for the Fenton reaction. Acidification of the tumor microenvironment also increases apoptosis resistance and enhances the invasiveness of tumors, thereby contributing to metastasis. ${ }^{98} 99$ Moreover, it was recently shown that the acidic environment drives a stem celllike phenotype in melanoma cells and thereby increases therapy resistance. ${ }^{100}$ Overall, a more acidic tumor microenvironment promotes cell death resistance. Therefore, it contributes to therapy resistance and increases the metastatic potential of tumors. However, it was recently reported that in tumor acidosis, long-chain PUFAs not present inside lipid droplets can be peroxidized, leading to increased ferroptosis. ${ }^{101}$ Use of diacylglycerol O-acyltransferase (DGAT) to inhibit lipid droplet formation resulted in the presence of more long-chain PUFAs available for peroxidation, and an antitumor effect in mice was observed due to ferroptotic cell death induction in cancer cells. It was discovered that acidosis causes autocrine transforming growth factor (TGF) $\beta$ secretion leading to enhanced lipid droplet formation. ${ }^{102}$ Interestingly, TGF- $\beta$ also caused lipid droplet formation in DCs, resulting in DC dysfunction and less effective immune responses. ${ }^{103}$ By blocking both TGF- $\beta$ and DGAT, DC activity recovered, demonstrating an efficient antitumor response.

\section{Ferroptosis in spheroids: challenges and new opportunities}

Ferroptosis and cell death modalities in general are still studied mostly in two-dimensional models (cells growing on a flat surface). However, this does not fully resemble the three-dimensional (3D) in vivo situation, where cells are often less responsive to cell death induction therapy. 3D models, such as spheroids, resemble the in vivo situation more closely because the cells produce ECM, which acts as an extra barrier for drugs to cross in order to induce tumor cell death, ${ }^{104-106}$ and more cell-to-cell contacts are present as well as cell-to-ECM contacts. Therefore, there has been a shift in recent years towards more relevant 3D models, including cancer spheroids. ${ }^{104} 107$ However, the more complex 3D models need more sophisticated equipment for analysis of cell death. ${ }^{104}$ Different cell types need to be distinguished, which still requires flow cytometry analysis or confocal microscopy. Recently, a new method has been developed that allows analysis of cell death in intact spheroids without the need to destroy the $3 \mathrm{D}$ structure. ${ }^{104}$

It was recently shown by using spheroids composed of human non-small cell lung tumor cells that the inner cells of the spheroids require nuclear factor erythroid 2-related factor 2 (NRF2) for their survival (figure 4). ${ }^{108}$ NRF2 is a basic leucine zipper (bZIP) protein that may regulate the expression of the antioxidant proteins protecting against the oxidative damage triggered by injury and inflammation. ${ }^{109}$ NRF2 and GPX4 are presumably needed to protect the cancer cells from ROS generation, which is toxic for the cells inside the spheroids and tumors. By inhibiting the different antioxidant pathways, this protection is circumvented and an overload of ROS in cancer might lead to efficient ferroptosis induction, eliminating all cancer cells (table 1 ).

Moreover, the compactness and stiffness of spheroids resemble the in vivo hypoxic tumor microenvironment, ${ }^{110}$ which can cause drug resistance through cell cycle arrest and downregulation of procell death proteins (eg, caspase-3 in apoptosis). ${ }^{111}$ Hypoxia is an important factor that might affect cellular responses to anticancer therapy by modulating the cell death responses. Hypoxia and lactate (discussed previously) are strongly correlated, as hypoxia can cause cancer cells to switch to glycolysis in order to survive. ${ }^{91}$ In spheroids, a hypoxic core might lead to upregulation of the hypoxia-inducible factor pathway (figure 4). In this regard, hypoxia-inducible factor- $1 \alpha$ can cause overexpression of membrane-bound carbonic anhydrase $9 .{ }^{112}$ Carbonic anhydrase nine causes $\mathrm{CO}_{2}$ to be converted to $\mathrm{H}^{+}$and $\mathrm{HCO}_{3}{ }^{-}$in order to regulate the extracellular $\mathrm{pH}$ for better cellular metabolism, but this creates 


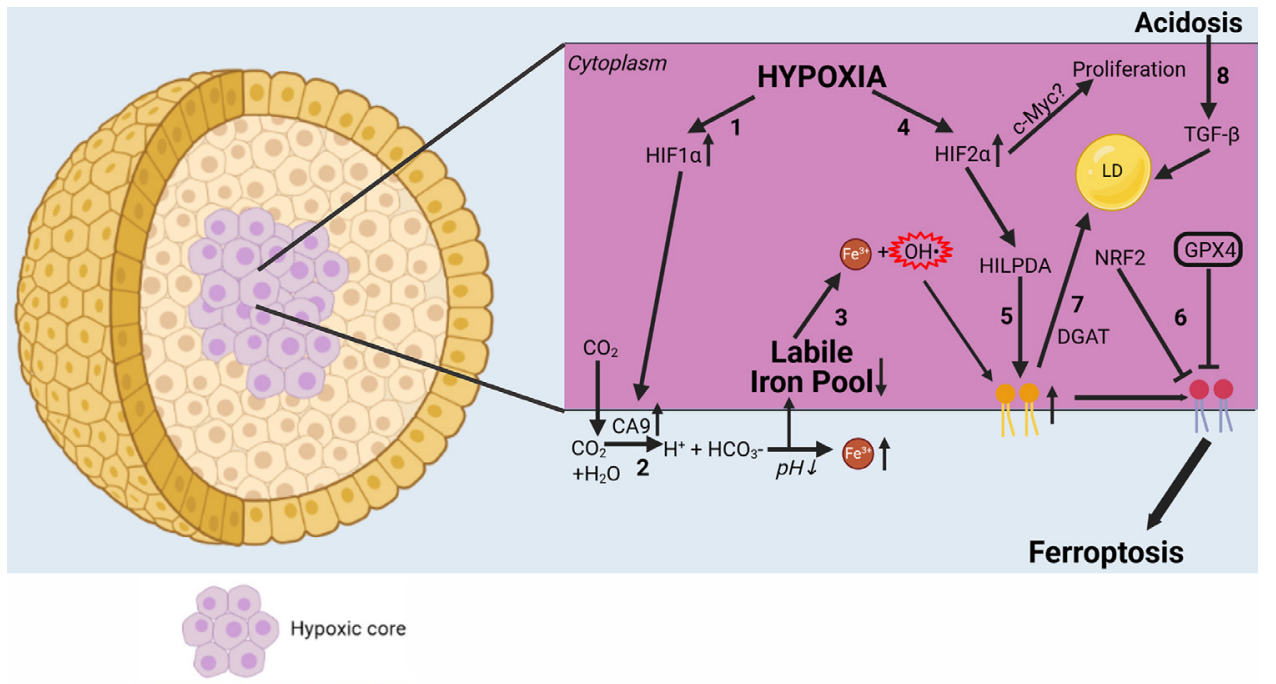

Figure 4 Complex interplay of ferroptosis with the tumor microenvironment. Hypoxia has an important influence on ferroptosis. (1) HIF-1 $\alpha$ is upregulated in hypoxic conditions. (2) In turn, HIF-1 $\alpha$ causes CA9 to be upregulated. ${ }^{114}$ CA9 then converts $\mathrm{CO}_{2}$ to $\mathrm{H}^{+}$and $\mathrm{HCO}_{3}$-, further acidifying the extracellular environment and leading to increased iron solubility. (3) Increased extracellular iron leads to a decrease in the labile iron pool via increased intracellular iron storage. (4) HIF-2 $\alpha$ is also upregulated in hypoxia, leading to stronger expression of HILPDA. ${ }^{118}$ (5) HILPDA then selectively enriches PUFAs in the plasma membrane, creating more substrate for lipid peroxidation. (6) In contrast, NRF2 and GPX4 protect cancer cells from ferroptosis by blocking lipid peroxidation. ${ }^{108}$ Due to hypoxia, many cancer types become dependent on GPX4 for their survival. (7) However, PUFAs can be stored in LDS by DGAT, making peroxidation impossible and thereby reducing sensitivity to ferroptosis. (8) Acidosis causes autocrine TGF- $\beta$ secretion, leading to increased LD formation. CA9, carbonic anhydrase 9; DGAT, diacylglycerol O-acyltransferase; GPX4, glutathione peroxidase 4; HIF1 $\alpha$, hypoxia-inducible factor $1 \alpha$; HIF2 $\alpha$, hypoxiainducible factor $2 \alpha$; HILPDA, hypoxia-inducible, lipid droplet-associated protein; NRF2, nuclear factor erythroid 2-related factor 2; LD, lipid droplet; oxPE, oxidized phosphatidylethanolamine; PUFA, polyunsaturated fatty acid.

an opportunity for cancer cells to resist ferroptosis. On one hand, this lowered extracellular $\mathrm{pH}$ might increase soluble iron in the blood, which results in decreased iron intake and increased iron storage. Since cancer cells are in a constant state of oxidative stress, they might become less susceptible to ferroptosis by increasing iron storage in the cellular compartments, ${ }^{113}$ thereby reducing the labile iron pool (figure 4), generating less free radicals, and decreasing cell death via the Fenton reaction occurring in ferroptosis. ${ }^{9}$ By mimicking this oxidative stress using cancer spheroids, better models can be obtained to study ferroptosis and screen for novel ferroptotic cell death inducers. However, on the other hand, it has already been observed that in some tumors, cancer cells become dependent on GPX4 for their survival. ${ }^{11415}$ Due to intracellular iron, lipid peroxidation could occur, but this is prevented by GPX4. Lipid peroxidation normally occurs at a slow rate because PUFAs are required to react with the free radicals generated by iron. ${ }^{3}$ In GPX4-dependent cancers, however, lipid peroxidation occurs frequently. This abundance of lipid peroxides formation, needed for ferroptosis execution, is generated by another protein of the HIF pathway, namely, HIF-2 $\alpha$ (figure 4). HIF-2 $\alpha$ is needed for survival of cancer cells in hypoxia, but this protein also creates a vulnerability for ferroptosis. ${ }^{116}$ In renal clear-cell carcinomas, HIF-2 $\alpha$ selectively enriches PUFAs, the rate-limiting substrates for lipid peroxidation and thus for ferroptosis, by activating the expression of hypoxia-inducible, lipid droplet-associated protein. ${ }^{116}$
Since GPX4 is still present inside the cancer cells, GPX4 keeps the process of lipid peroxidation in check. This makes the these cancers dependent on GPX4 for survival. By targeting this enzyme, ferroptosis might be induced more easily. Therefore, targeting GPX4 might lead to more effective cell death induction in cancer.

However, HIF-2 $\alpha$ also promotes cell-cycle progression in hypoxic cells, which correlates with enhanced c-Myc promoter binding. Enhanced c-Myc activity likely contributes to HIF-2 $\alpha$-mediated neoplastic progression following loss of the von Hippel-Lindau tumor suppressor and influences the behavior of hypoxic tumor cells. ${ }^{117}$ This suggests that HIF-2 $\alpha$ might actually promote cancer cell proliferation in hypoxia. Also, HIF- $2 \alpha$ modulates lipid storage to sustain ER homeostasis, particularly under conditions of nutrient and oxygen limitation, thereby enhancing tumor cell survival. ${ }^{118}$ Hence, HIF-2 $\alpha$ might have different effects on the outcome of ferroptosis treatment. In addition, hypoxia provides a tumor microenvironment that allows selection of cell death-resistant cell types. ${ }^{119}$ It will be interesting to evaluate how both the protective signals and the intrinsic sensitivity to cell death in cancer affect cancer treatment efficiency.

In conclusion, it is important to keep in mind that in $3 \mathrm{D}$ models, including spheroids, many factors can influence each other (eg, cell adhesion, stiffness, concentration gradient, hypoxia, etc), which will have an impact on the overall resistance to ferroptotic cell death and its immunogenicity, and thus to therapy. Nonetheless, 3D models 
are more reliable for studying cell death responses to cancer therapy, which might require a multidisciplinary approach combining different methods to obtain a complete view of cell death in a three-dimensional setting.

\section{FUTURE PERSPECTIVES}

The use of ferroptosis in cancer therapy has proven to be an interesting alternative to targeting apoptosis and necroptosis. However, more research is needed to uncover the full therapeutic potential of ferroptosis (figure 5 and table 1). For example, the role of blebs released during ferroptosis is currently uncertain. ${ }^{18}{ }^{22}$ It would be interesting to investigate the content of those blebs and their function in immunogenicity. For example, it is known that apoptotic bodies contain fragmented DNA, mitochondria and T-cell antigens, ${ }^{21}{ }^{120}$ but uptake of apoptotic bodies leads to immunosuppression. ${ }^{121}$ Whether this is also true for ferroptotic blebs is not known and requires further investigation. Also, the surface expression pattern of CRT during ferroptosis has only been shown for ferroptosislike cell death, but it is unknown if ferroptosis-inducing drugs such as RSL3 promote CRT exposure. ${ }^{65} 81$ Though HMGB $^{6566}$ and $\mathrm{ATP}^{65}$ release from ferroptotic cancer cells has been proven, it is important to discover the full repertoire of DAMPs released during ferroptosis in order to investigate the full immunogenic potential of this cell death pathway. Furthermore, the question of ferroptosis time span and its relevance to the immunogenic properties of ferroptosis has been raised. ${ }^{65}$ Early ferroptosis has been shown to be the most efficient in terms of induction of immunogenicity, when most HMGB1 and ATP are released, generating the best adjuvant effect, early ferroptotic cancer cells activated BMDCs in vitro and protected mice against tumor growth. Some ferroptosisinducing treatments, such as sulfasalazine, sorafenib and erastin, have shown great potential in preclinical models (table 1). However, mainly immunodeficient mice were used, and the important influence of the immune system was ignored. It is necessary to investigate the therapeutic efficacy of ferroptosis induction in immunocompetent mice. Also, samples from patients were treated with ferroptosis-inducing agents, although no clinical trials focusing on ferroptosis have been performed so far. Overall, it remains too early to draw conclusions about therapeutic efficacy from the preclinical research. Clinical studies are needed to determine the therapeutic value of ferroptosis induction in patients with different types of cancers.

The role of oxPL in the immunogenicity of ferroptosis still remains poorly investigated, although they represent considerable part of ferroptotic cell death. Furthermore, the important element of immunogenicity, antigenicity, that is, TAA and neoantigens of cancer cells, has not yet been demonstrated for ferroptosis, which might open up other possibilities for anti-cancer immunotherapies. It is important to consider the tumor type, tumor microenvironment and genetic background of the patient when

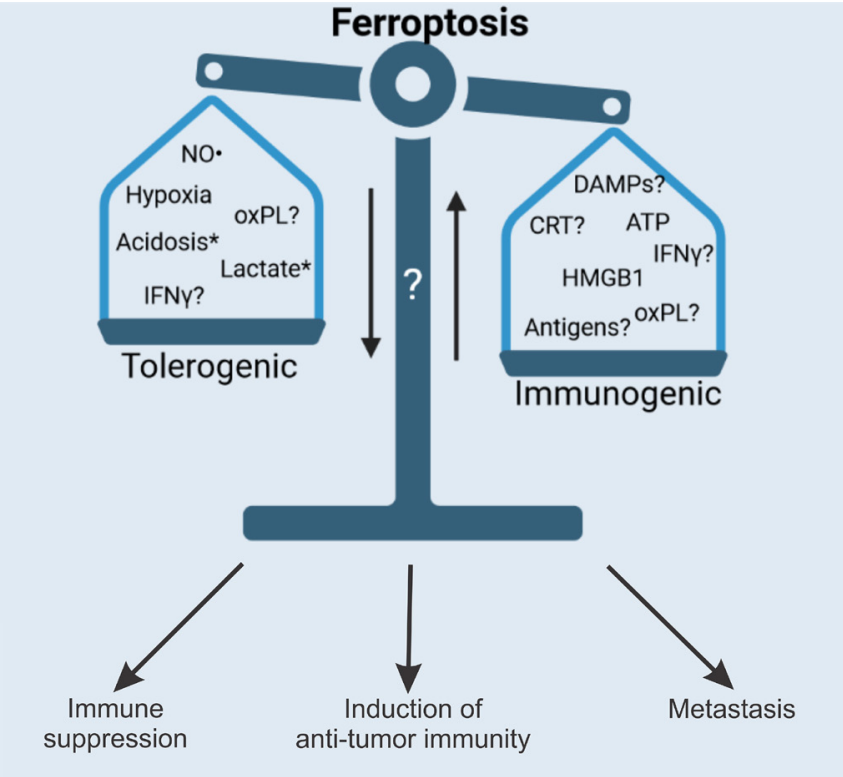

Figure 5 Future perspectives. Ferroptosis has proven to be an attractive alternative to apoptosis and necroptosis for use in cancer therapy. However, more research is needed to understand the full therapeutic potential of ferroptosis. The full repertoire of spatiotemporal release of both DAMPs and cytokines (ie, iDAMPs) needs to be investigated and linked to the immunogenic potential of ferroptosis. As ferroptotic cancer cells contain large numbers of various oxidized and peroxidized lipids and PLs, it is possible to speculate about their effects on the anticancer immune response. Nevertheless, studies are needed to clarify whether ferroptosis-derived oxPLs are responsible for modulating ferroptosis immunogenicity, which may explain the nonimmunogenicity of the later stages of ferroptotic cell death. ${ }^{65}$ Importantly, the modulation of antigenicity by ferroptosis is still poorly understood, and there is a need to also investigate the formation of memory $\mathrm{T}$ cells, which is an important contributor to the therapeutic potential of ferroptosis. In addition, IFN- $\gamma$ released from $\mathrm{CD}^{+} \mathrm{T}$ cells has been reported to promote cancer cell lipid peroxidation and ferroptosis. ${ }^{92}$. in addition, expression of the protumor PDL1 is increased on tumor cells due to increased secretion of IFN- $\gamma$ by $C D 8^{+} T$ cells. ${ }^{47}$ these findings might also explain the tolerogenic (ie, immune suppressive) phenotype of late ferroptotic cells reported in Efimova et al. ${ }^{65}$ Finally, it is important to understand how ferroptotic cancer cells affect metastatic capacity. *Lactate and acidification do not have a direct effect on immunogenicity (or it is not known yet), but they increase cell death resistance, thereby reducing therapeutic efficacy. CRT, calreticulin; DAMP, damage-associated molecular pattern; HMGB1, high-mobility group 1; iDAMP, inducible danger-associated molecular pattern; IFN- $\gamma$, interferon gamma; NO•, nitric oxide; oxPL, oxidized phospholipid; PDL1, prodeath ligand 1; PL, phospholipid.

choosing the optimal personalized therapy because different tumors might generate an altered response during ferroptosis. ${ }^{122}$ Therefore, it is important to investigate the role of the microenvironment in ferroptosis in different tissues. In some organs, ferroptosis induction might lead to an exacerbated inflammatory loop that promotes tumors and thereby limits therapy efficiency. 
The microenvironment can have an enormous influence on cell death and immunogenicity either by increasing resistance or enhancing metastatic potential, which would promote escape from therapy ${ }^{99}{ }^{100}$ Understanding the complex interactions between different cell types within the tumor will be pivotal in investigating the immunogenic potential of ferroptosis.

\section{Twitter Robin Demuynck @RobinDemuynck and Dmitri V Krysko @DmitriKrysko}

Contributors RD and IE drafted the manuscript and the figures. FN drafted the table. DVK initiated, designed, and supervised the study and revised the manuscript.

Funding The CDIT Laboratory is supported by FWO-Flanders Research Grants (G043219N and G016221N) and by BOF (01/03618). Robin Demuynck (11E3121N) and luliia Efimova (11F7721N) are PhD students in the CDIT Laboratory, and they both hold PhD fellowships from FWO-Flanders. Analysis of the literature on ferroptosis induction in anti-cancer therapy, including photodynamic therapy, is also supported by a grant from Russian Science Foundation (project no.1815-00279 https://rscf.ru/en/project/18-15-00279/). The figures were created with wwwBioRendercom.

Competing interests None declared.

Patient consent for publication Not applicable.

Provenance and peer review Not commissioned; externally peer reviewed.

Open access This is an open access article distributed in accordance with the Creative Commons Attribution Non Commercial (CC BY-NC 4.0) license, which permits others to distribute, remix, adapt, build upon this work non-commercially, and license their derivative works on different terms, provided the original work is properly cited, appropriate credit is given, any changes made indicated, and the use is non-commercial. See http://creativecommons.org/licenses/by-nc/4.0/.

\section{ORCID iDs}

Robin Demuynck http://orcid.org/0000-0001-7930-8347

Iuliia Efimova http://orcid.org/0000-0001-7795-7490

Dmitri V Krysko http://orcid.org/0000-0002-9692-2047

\section{REFERENCES}

1 Dixon SJ, Lemberg KM, Lamprecht MR, et al. Ferroptosis: an irondependent form of nonapoptotic cell death. Cell 2012;149:1060-72.

2 Ingold I, Berndt C, Schmitt S, et al. Selenium utilization by GPX4 is required to prevent hydroperoxide-induced ferroptosis. Cell 2018;172:409-22.

3 D'Herde K, Krysko DV. Ferroptosis: oxidized PEs trigger death. Nat Chem Biol 2017;13:4-5.

4 Friedmann Angeli JP, Krysko DV, Conrad M. Ferroptosis at the crossroads of cancer-acquired drug resistance and immune evasion. Nat Rev Cancer 2019;19:405-14.

5 Kagan VE, Mao G, Qu F, et al. Oxidized arachidonic and adrenic PEs navigate cells to ferroptosis. Nat Chem Biol 2017;13:81-90.

6 Agmon E, Solon J, Bassereau P, et al. Modeling the effects of lipid peroxidation during ferroptosis on membrane properties. Sci Rep 2018;8:5155.

7 Yang WS, SriRamaratnam R, Welsch ME, et al. Regulation of ferroptotic cancer cell death by GPX4. Cell 2014;156:317-31.

8 Dixon SJ, Stockwell BR. The role of iron and reactive oxygen species in cell death. Nat Chem Biol 2014;10:9-17.

9 Mishchenko TA, Balalaeva IV, Vedunova MV, et al. Ferroptosis and photodynamic therapy synergism: enhancing anticancer treatment. Trends Cancer 2021;7:484-7.

10 Anthonymuthu TS, Tyurina YY, Sun W-Y, et al. Resolving the paradox of ferroptotic cell death: Ferrostatin-1 binds to 15LOX/ PEBP1 complex, suppresses generation of peroxidized ETE-PE, and protects against ferroptosis. Redox Biol 2021;38:101744.

11 Wenzel SE, Tyurina YY, Zhao J, et al. PEBP1 Wardens ferroptosis by enabling lipoxygenase generation of lipid death signals. Cell 2017; 171:628-41.

12 Doll S, Proneth B, Tyurina YY, et al. Acsl4 dictates ferroptosis sensitivity by shaping cellular lipid composition. Nat Chem Biol 2017;13:91-8.

13 Tang D, Kroemer G. Peroxisome: the new player in ferroptosis. Signal Transduct Target Ther 2020;5:273.
14 Cui W, Liu D, Gu W, et al. Peroxisome-driven ether-linked phospholipids biosynthesis is essential for ferroptosis. Cell Death Differ 2021;28:2536-51.

15 Zou Y, Henry WS, Ricq EL, et al. Plasticity of ether lipids promotes ferroptosis susceptibility and evasion. Nature 2020;585:603-8.

16 Xie Y, Hou W, Song X, et al. Ferroptosis: process and function. Cell Death Differ 2016;23:369-79.

17 Orchel A, Chodurek E, Jaworska-Kik M, et al. Anticancer activity of the acetylenic derivative of Betulin phosphate involves induction of necrotic-like death in breast cancer cells in vitro. Molecules 2021;26:615.

18 Van der Meeren L, Verduijn J, Krysko DV, et al. Afm analysis enables differentiation between apoptosis, necroptosis, and ferroptosis in murine cancer cells. iScience2020;23:101816.

19 Krysko DV, Brouckaert G, Kalai M, et al. Mechanisms of internalization of apoptotic and necrotic L929 cells by a macrophage cell line studied by electron microscopy. J Morphol 2003;258:336-45

20 Vandenabeele P, Galluzzi L, Vanden Berghe T, et al. Molecular mechanisms of necroptosis: an ordered cellular explosion. Nat Rev Mol Cell Biol 2010;11:700-14.

21 Krysko DV, Denecker G, Festjens N, et al. Macrophages use different internalization mechanisms to clear apoptotic and necrotic cells. Cell Death Differ 2006;13:2011-22.

22 Kajiwara K, Beharier O, Chng C-P, et al. Ferroptosis induces membrane blebbing in placental trophoblasts. J Cell Sci 2022;135:jcs255737.

23 Tang HM, Tang HL. Cell recovery by reversal of ferroptosis. Biol Open 2019;8:bio043182.

24 Hiller S, Broz P. Active membrane rupture spurs a range of cell deaths. Nature 2021;591:36-7.

25 Kayagaki N, Kornfeld OS, Lee BL, et al. NINJ1 mediates plasma membrane rupture during lytic cell death. Nature 2021;591:131-6.

26 Riegman M, Sagie L, Galed C, et al. Ferroptosis occurs through an osmotic mechanism and propagates independently of cell rupture. Nat Cell Biol 2020;22:1042-8.

27 Pedrera L, Espiritu RA, Ros U, et al. Ferroptotic pores induce $\mathrm{Ca}^{2+}$ fluxes and ESCRT-III activation to modulate cell death kinetics. Cell Death Differ 2021;28:1-14.

28 Verduijn J, Van der Meeren L, Krysko DV, et al. Deep learning with digital holographic microscopy discriminates apoptosis and necroptosis. Cell Death Discov 2021;7:229.

29 Matzinger P. An innate sense of danger. Semin Immunol 1998:10:399-415.

30 Tang D, Kang R, Coyne CB, et al. Pamps and DAMPs: signal 0 s that Spur autophagy and immunity. Immunol Rev 2012;249:158-75.

31 Krysko DV, Garg AD, Kaczmarek A, et al. Immunogenic cell death and DAMPs in cancer therapy. Nat Rev Cancer 2012;12:860-75.

32 Galluzzi L, Buqué A, Kepp O, et al. Immunogenic cell death in cancer and infectious disease. Nat Rev Immunol 2017;17:97-111.

33 Galluzzi L, Vitale I, Warren S, et al. Consensus guidelines for the definition, detection and interpretation of immunogenic cell death. $J$ Immunother Cancer 2020;8:e000337.

34 Krysko DV, D'Herde K, Vandenabeele P. Clearance of apoptotic and necrotic cells and its immunological consequences. Apoptosis 2006;11:1709-26.

35 Casares N, Pequignot MO, Tesniere A, et al. Caspase-Dependent immunogenicity of doxorubicin-induced tumor cell death. J Exp Med 2005;202:1691-701.

36 Sun Y, Yin E, Tan Y, et al. Immunogenicity and cytotoxicity of a platinum(IV) complex derived from capsaicin. Dalton Trans 2021;50:3516-22.

37 Turubanova VD, Balalaeva IV, Mishchenko TA, et al. Immunogenic cell death induced by a new photodynamic therapy based on photosens and photodithazine. J Immunother Cancer 2019;7:350.

38 Mishchenko TA, Turubanova VD, Mitroshina EV, et al. Effect of novel porphyrazine photosensitizers on normal and tumor brain cells. $J$ Biophotonics 2020;13:e201960077.

39 Apetoh L, Ghiringhelli F, Tesniere A, et al. Toll-Like receptor 4-dependent contribution of the immune system to anticancer chemotherapy and radiotherapy. Nat Med 2007;13:1050-9.

40 Obeid M, Tesniere A, Ghiringhelli F, et al. Calreticulin exposure dictates the immunogenicity of cancer cell death. Nat Med 2007;13:54-61.

41 Garg AD, Krysko DV, Verfaillie T, et al. A novel pathway combining calreticulin exposure and ATP secretion in immunogenic cancer cell death. Embo J 2012;31:1062-79.

42 Murai S, Yamaguchi Y, Shirasaki Y, et al. A FRET biosensor for necroptosis uncovers two different modes of the release of DAMPs. Nat Commun 2018;9:4457. 
43 Aaes TL, Kaczmarek A, Delvaeye T, et al. Vaccination with Necroptotic cancer cells induces efficient anti-tumor immunity. Cell Rep 2016;15:274-87.

44 Hanahan D, Weinberg RA. Hallmarks of cancer: the next generation Cell 2011;144:646-74.

45 Zhang J-M, An J. Cytokines, inflammation, and pain. Int Anesthesiol Clin 2007;45:27-37.

46 Timmermans K, Kox M, Vaneker M, et al. Plasma levels of danger-associated molecular patterns are associated with immune suppression in trauma patients. Intensive Care Med 2016;42:551-61.

47 Abiko K, Matsumura N, Hamanishi J, et al. IFN- $\gamma$ from lymphocytes induces PD-L1 expression and promotes progression of ovarian cancer. Br J Cancer 2015;112:1501-9.

48 Schroder K, Hertzog PJ, Ravasi T, et al. Interferon-Gamma: an overview of signals, mechanisms and functions. J Leukoc Biol 2004;75:163-89.

49 Workenhe ST, Nguyen A, Bakhshinyan D, et al. De novo necroptosis creates an inflammatory environment mediating tumor susceptibility to immune checkpoint inhibitors. Commun Biol 2020;3:645

50 Yatim N, Cullen S, Albert ML. Dying cells actively regulate adaptive immune responses. Nat Rev Immunol 2017;17:262-75.

51 Yatim N, Jusforgues-Saklani H, Orozco S, et al. RIPK1 and NF- $\kappa B$ signaling in dying cells determines cross-priming of $\mathrm{CD}^{+} \mathrm{T}$ cells. Science 2015;350:328-34.

52 Sistigu A, Yamazaki T, Vacchelli E, et al. Cancer cell-autonomous contribution of type I interferon signaling to the efficacy of chemotherapy. Nat Med 2014;20:1301-9.

53 Orozco SL, Daniels BP, Yatim N, et al. RIPK3 activation leads to cytokine synthesis that continues after loss of cell membrane integrity. Cell Rep 2019;28:2275-87.

54 Brouckaert G, Kalai M, Krysko DV, et al. Phagocytosis of necrotic cells by macrophages is phosphatidylserine dependent and does not induce inflammatory cytokine production. Mol Biol Cell 2004;15:1089-100.

55 Zhang Y, Cheung Y-K, Ng DKP, et al. Immunogenic necroptosis in the anti-tumor photodynamic action of BAM-SiPc, a silicon(IV) phthalocyanine-based photosensitizer. Cancer Immunol Immunother 2021;70:485-95.

$56 \mathrm{Li} \mathrm{X}$, Zheng J, Chen S, et al. Oleandrin, a cardiac glycoside, induces immunogenic cell death via the PERK/elF2 $\alpha /$ ATF4/CHOP pathway in breast cancer. Cell Death Dis 2021;12:314

57 Dhatchinamoorthy K, Colbert JD, Rock KL. Cancer immune evasion through loss of MHC class I antigen presentation. Front Immunol 2021;12:469.

58 Jhunjhunwala S, Hammer C, Delamarre L. Antigen presentation in cancer: insights into tumour immunogenicity and immune evasion. Nat Rev Cancer 2021;21:298-312.

59 Zhao Y, Baldin AV, Isayev O, et al. Cancer vaccines: antigen selection strategy. Vaccines 2021;9. doi:10.3390/vaccines9020085. [Epub ahead of print: 25012021 ]

60 Ning F, Cole CB, Annunziata CM. Driving immune responses in the ovarian tumor microenvironment. Front Oncol 2020;10:604084.

61 Lokhov PG, Balashova EE. Antigenic essence: upgrade of cellular cancer vaccines. Cancers 2021;13. doi:10.3390/cancers13040774. [Epub ahead of print: 1202 2021].

62 Khong HT, Restifo NP. Natural selection of tumor variants in the generation of "tumor escape" phenotypes. Nat Immunol 2002;3:999-1005.

63 Aaes TL, Vandenabeele P. The intrinsic immunogenic properties of cancer cell lines, immunogenic cell death, and how these influence host antitumor immune responses. Cell Death Differ 2021;28:843-60.

64 Aaes TL, Verschuere H, Kaczmarek A, et al. Immunodominant AH1 Antigen-Deficient Necroptotic, but not apoptotic, murine cancer cells induce antitumor protection. J.i. 2020;204:775-87.

65 Efimova I, Catanzaro E, Van der Meeren L, et al. Vaccination with early ferroptotic cancer cells induces efficient antitumor immunity. $J$ Immunother Cancer 2020;8:e001369.

66 Wen Q, Liu J, Kang R, et al. The release and activity of HMGB1 in ferroptosis. Biochem Biophys Res Commun 2019;510:278-83.

67 Miyake S, Murai S, Kakuta S, et al. Identification of the hallmarks of necroptosis and ferroptosis by transmission electron microscopy. Biochem Biophys Res Commun 2020;527:839-44.

68 Li Y, Feng D, Wang Z, et al. Ischemia-Induced ACSI4 activation contributes to ferroptosis-mediated tissue injury in intestinal ischemia/reperfusion. Cell Death Differ 2019;26:2284-99.

69 Li W, Feng G, Gauthier JM, et al. Ferroptotic cell death and TLR4/Trif signaling initiate neutrophil recruitment after heart transplantation. J Clin Invest 2019;129:2293-304.
70 Linkermann A, Skouta R, Himmerkus N, et al. Synchronized renal tubular cell death involves ferroptosis. Proc Natl Acad Sci U S A 2014;111:16836-41.

71 Wang Y, Chen Q, Shi C, et al. Mechanism of glycyrrhizin on ferroptosis during acute liver failure by inhibiting oxidative stress. Mol Med Rep 2019;20:4081-90.

72 Ye F, Chai W, Xie M, et al. HMGB1 regulates erastin-induced ferroptosis via RAS-JNK/p38 signaling in HL-60/NRAS ${ }^{\text {661 }}$ cells. Am $J$ Cancer Res 2019:9:730-9.

73 Huttunen HJ, Fages C, Kuja-Panula J, et al. Receptor for advanced glycation end products-binding $\mathrm{COOH}$-terminal motif of amphoterin inhibits invasive migration and metastasis. Cancer Res 2002;62:4805-11.

74 Park JS, Svetkauskaite D, He Q, et al. Involvement of Toll-like receptors 2 and 4 in cellular activation by high mobility group box 1 protein. J Biol Chem 2004;279:7370-7.

75 Apetoh L, Ghiringhelli F, Tesniere A, et al. The interaction between HMGB1 and TLR4 dictates the outcome of anticancer chemotherapy and radiotherapy. Immunol Rev 2007;220:47-59.

76 Guerriero JL, Ditsworth D, Catanzaro JM, et al. Dna alkylating therapy induces tumor regression through an HMGB1-mediated activation of innate immunity. J Immunol 2011;186:3517-26.

77 Yamazaki T, Hannani D, Poirier-Colame V, et al. Defective immunogenic cell death of HMGB1-deficient tumors: compensatory therapy with TLR4 agonists. Cell Death Differ 2014;21:69-78.

78 Mariathasan S, Weiss DS, Newton K, et al. Cryopyrin activates the inflammasome in response to toxins and ATP. Nature 2006;440:228-32.

79 Ghiringhelli F, Apetoh L, Tesniere A, et al. Activation of the NLRP3 inflammasome in dendritic cells induces IL-1beta-dependent adaptive immunity against tumors. Nat Med 2009;15:1170-8.

80 Elliott MR, Chekeni FB, Trampont PC, et al. Nucleotides released by apoptotic cells act as a find-me signal to promote phagocytic clearance. Nature 2009;461:282-6.

81 Yu B, Choi B, Li W, et al. Magnetic field boosted ferroptosis-like cell death and responsive MRI using hybrid vesicles for cancer immunotherapy. Nat Commun 2020;11:3637.

82 O'Donnell VB, Aldrovandi M, Murphy RC, et al. Enzymatically oxidized phospholipids assume center stage as essential regulators of innate immunity and cell death. Sci Signal 2019;12:eaau2293.

83 Friedmann Angeli JP, Schneider M, Proneth B, et al. Inactivation of the ferroptosis regulator GPX4 triggers acute renal failure in mice. Nat Cell Biol 2014;16:1180-91.

84 Stockwell BR, Friedmann Angeli JP, Bayir H, et al. Ferroptosis: a regulated cell death nexus linking metabolism, redox biology, and disease. Cell 2017;171:273-85.

85 Tyurin VA, Balasubramanian K, Winnica D, et al. Oxidatively modified phosphatidylserines on the surface of apoptotic cells are essential phagocytic 'eat-me' signals: cleavage and inhibition of phagocytosis by Lp-PLA2. Cell Death Differ 2014;21:825-35.

86 Greenberg ME, Sun M, Zhang R, et al. Oxidized phosphatidylserineCD36 interactions play an essential role in macrophage-dependent phagocytosis of apoptotic cells. J Exp Med 2006;203:2613-25.

87 Uderhardt S, Herrmann M, Oskolkova OV, et al. 12/15-Lipoxygenase orchestrates the clearance of apoptotic cells and maintains immunologic tolerance. Immunity 2012;36:834-46.

88 Klöditz K, Fadeel B. Three cell deaths and a funeral: macrophage clearance of cells undergoing distinct modes of cell death. Cell Death Discov 2019;5:65.

89 Veglia F, Tyurin VA, Mohammadyani D, et al. Lipid bodies containing oxidatively truncated lipids block antigen cross-presentation by dendritic cells in cancer. Nat Commun 2017;8:2122.

90 Ramakrishnan R, Tyurin VA, Tuyrin VA, et al. Oxidized lipids block antigen cross-presentation by dendritic cells in cancer. $\mathrm{J}$ Immuno 2014;192:2920-31

91 Ugolini A, Tyurin VA, Tyurina YY, et al. Polymorphonuclear myeloidderived suppressor cells limit antigen cross-presentation by dendritic cells in cancer. JCI Insight 2020;5:e138581.

92 Wang W, Green M, Choi JE, et al. CD8 ${ }^{+} \mathrm{T}$ cells regulate tumour ferroptosis during cancer immunotherapy. Nature 2019;569:270-4.

93 Vitale I, Shema E, Loi S, et al. Intratumoral heterogeneity in cancer progression and response to immunotherapy. Nat Med 2021;27:212-24.

94 Kapralov AA, Yang Q, Dar HH, et al. Redox lipid reprogramming commands susceptibility of macrophages and microglia to ferroptotic death. Nat Chem Biol 2020;16:278-90.

95 Dai E, Han L, Liu J, et al. Autophagy-Dependent ferroptosis drives tumor-associated macrophage polarization via release and uptake of oncogenic KRAS protein. Autophagy 2020;16:2069-83. 
96 Brizel DM, Schroeder T, Scher RL, et al. Elevated tumor lactate concentrations predict for an increased risk of metastases in headand-neck cancer. Int J Radiat Oncol Biol Phys 2001;51:349-53.

97 Zhao Y, Li M, Yao X, et al. HCAR1/MCT1 regulates tumor ferroptosis through the lactate-mediated AMPK-SCD1 activity and its therapeutic implications. Cell Rep 2020;33:108487.

98 Peppicelli S, Bianchini F, Torre E, et al. Contribution of acidic melanoma cells undergoing epithelial-to-mesenchymal transition to aggressiveness of non-acidic melanoma cells. Clin Exp Metastasis 2014;31:423-33.

99 Damaghi M, Gillies R. Phenotypic changes of acid-adapted cancer cells push them toward aggressiveness in their evolution in the tumor microenvironment. Cell Cycle 2017;16:1739-43.

100 Andreucci E, Peppicelli S, Ruzzolini J, et al. The acidic tumor microenvironment drives a stem-like phenotype in melanoma cells. J Mol Med 2020;98:1431-46.

101 Dierge E, Debock E, Guilbaud C, et al. Peroxidation of n-3 and n-6 polyunsaturated fatty acids in the acidic tumor environment leads to ferroptosis-mediated anticancer effects. Cell Metab 2021:33:1701-15

102 Corbet C, Bastien E, Santiago de Jesus JP, et al. TGF $\beta 2$-induced formation of lipid droplets supports acidosis-driven EMT and the metastatic spreading of cancer cells. Nat Commun 2020;11:454.

103 Trempolec N, Degavre C, Doix B, et al. Acidosis-Induced TGF- $\beta 2$ production promotes lipid droplet formation in dendritic cells and alters their potential to support anti-mesothelioma T cell response. Cancers 2020;12:1284.

104 Demuynck R, Efimova I, Lin A, et al. A 3D cell death assay to quantitatively determine ferroptosis in spheroids. Cells 2020;9:703.

105 Cui X, Hartanto Y, Zhang H. Advances in multicellular spheroids formation. J R Soc Interface 2017;14:20160877.

106 McKenzie AJ, Hicks SR, Svec KV, et al. The mechanical microenvironment regulates ovarian cancer cell morphology, migration, and spheroid disaggregation. Sci Rep 2018;8:7228

107 Vande Voorde J, Ackermann T, Pfetzer N, et al. Improving the metabolic fidelity of cancer models with a physiological cell culture medium. Sci Adv 2019;5:eaau7314.

108 Takahashi N, Cho P, Selfors LM, et al. 3D culture models with CRISPR screens reveal hyperactive Nrf2 as a prerequisite for spheroid formation via regulation of proliferation and ferroptosis. Mol Cell 2020;80:828-44.

109 Ndlovu S, Nagiah S, Abdul NS, et al. Deoxynivalenol downregulates NRF2-induced cytoprotective response in human hepatocellular carcinoma (HepG2) cells. Toxicon 2021;193:4-12.

110 Leek R, Grimes DR, Harris AL, et al. Methods: using threedimensional culture (spheroids) as an in vitro model of tumour hypoxia. Adv Exp Med Biol 2016;899:167-96.

111 Imamura Y, Mukohara T, Shimono Y, et al. Comparison of 2D- and 3D-culture models as drug-testing platforms in breast cancer. Oncol Rep 2015;33:1837-43

$112 \mathrm{Li}$ Z, Jiang L, Chew SH, et al. Carbonic anhydrase 9 confers resistance to ferroptosis/apoptosis in malignant mesothelioma under hypoxia. Redox Biol 2019;26:101297.

113 Sosa V, Moliné T, Somoza R, et al. Oxidative stress and cancer: an overview. Ageing Res Rev 2013;12:376-90.

114 Zou Y, Palte MJ, Deik AA, et al. A GPX4-dependent cancer cell state underlies the clear-cell morphology and confers sensitivity to ferroptosis. Nat Commun 2019;10:1617.

115 Viswanathan VS, Ryan MJ, Dhruv HD, et al. Dependency of a therapy-resistant state of cancer cells on a lipid peroxidase pathway. Nature 2017;547:453-7.

116 Zou Yet al. HIF-2 $\alpha$ drives an intrinsic vulnerability to ferroptosis in clear cell renal cell carcinoma. bioRxiv2018:388041.

117 Gordan JD, Bertout JA, Hu C-J, et al. Hif-2Alpha promotes hypoxic cell proliferation by enhancing c-myc transcriptional activity. Cancer Cell 2007;11:335-47.

118 Qiu B, Ackerman D, Sanchez DJ, et al. HIF2 $\alpha$-Dependent lipid storage promotes endoplasmic reticulum homeostasis in clear-cell renal cell carcinoma. Cancer Discov 2015;5:652-67.

119 Graeber TG, Osmanian C, Jacks T, et al. Hypoxia-mediated selection of cells with diminished apoptotic potential in solid tumours. Nature 1996;379:88-91.

120 György B, Szabó TG, Pásztói M, et al. Membrane vesicles, current state-of-the-art: emerging role of extracellular vesicles. Cell Mol Life Sci 2011;68:2667-88.

121 Savill J, Dransfield I, Gregory C, et al. A blast from the past: clearance of apoptotic cells regulates immune responses. Nat Rev Immunol 2002;2:965-75.

122 Dai E, Han L, Liu J, et al. Ferroptotic damage promotes pancreatic tumorigenesis through a TMEM173/STING-dependent DNA sensor pathway. Nat Commun 2020;11:6339.
123 Louandre C, Marcq I, Bouhlal H, et al. The retinoblastoma (Rb) protein regulates ferroptosis induced by sorafenib in human hepatocellular carcinoma cells. Cancer Lett 2015;356:971-7.

124 Li Z-J, Dai H-Q, Huang X-W, et al. Artesunate synergizes with sorafenib to induce ferroptosis in hepatocellular carcinoma. Acta Pharmacol Sin 2021;42:301-10.

125 Sun X, Ou Z, Chen R, et al. Activation of the p62-Keap1-NRF2 pathway protects against ferroptosis in hepatocellular carcinoma cells. Hepatology 2016;63:173-84.

126 Jin M, Shi C, Li T, et al. Solasonine promotes ferroptosis of hepatoma carcinoma cells via glutathione peroxidase 4-induced destruction of the glutathione redox system. Biomed Pharmacother 2020;129:110282

127 Wang Q, Bin C, Xue Q, et al. GSTZ1 sensitizes hepatocellular carcinoma cells to sorafenib-induced ferroptosis via inhibition of NRF2/GPX4 axis. Cell Death Dis 2021;12:1-16.

128 Sun X, Niu X, Chen R, et al. Metallothionein-1G facilitates sorafenib resistance through inhibition of ferroptosis. Hepatology 2016;64:488-500.

129 Sun J, Zhou C, Zhao Y, et al. Quiescin sulfhydryl oxidase 1 promotes sorafenib-induced ferroptosis in hepatocellular carcinoma by driving EGFR endosomal trafficking and inhibiting Nrf2 activation. Redox Biol 2021:41:101942.

130 Gao Z, Deng G, Li Y, et al. Actinidia chinensis Planch prevents proliferation and migration of gastric cancer associated with apoptosis, ferroptosis activation and mesenchymal phenotype suppression. Biomed Pharmacother 2020;126:110092.

$131 \mathrm{Hao} \mathrm{S,} \mathrm{Yu} \mathrm{J,} \mathrm{He} \mathrm{W,} \mathrm{et} \mathrm{al.} \mathrm{Cysteine} \mathrm{dioxygenase} 1$ mediates erastininduced ferroptosis in human gastric cancer cells. Neoplasia 2017; 19:1022-32.

132 Roh J-L, Kim EH, Jang H, et al. Nrf2 inhibition reverses the resistance of cisplatin-resistant head and neck cancer cells to artesunate-induced ferroptosis. Redox Biol 2017;11:254-62.

133 Roh J-L, Kim EH, Jang HJ, et al. Induction of ferroptotic cell death for overcoming cisplatin resistance of head and neck cancer. Cancer Lett 2016;381:96-103.

$134 \mathrm{Kim}$ EH, Shin D, Lee J, et al. Cisd2 inhibition overcomes resistance to sulfasalazine-induced ferroptotic cell death in head and neck cancer. Cancer Lett 2018;432:180-90.

135 Lee J, You JH, Shin D, et al. Inhibition of glutaredoxin 5 predisposes cisplatin-resistant head and neck cancer cells to ferroptosis. Theranostics 2020;10:7775.

136 You JH, Lee J, Roh J-L. Mitochondrial pyruvate carrier 1 regulates ferroptosis in drug-tolerant persister head and neck cancer cells via epithelial-mesenchymal transition. Cancer Lett 2021;507:40-54.

137 Zhang X, Sui S, Wang L, et al. Inhibition of tumor Propellant glutathione peroxidase 4 induces ferroptosis in cancer cells and enhances anticancer effect of cisplatin. $J$ Cell Physiol 2020;235:3425-37.

138 Chen P, Wu Q, Feng J, et al. Erianin, a novel dibenzyl compound in Dendrobium extract, inhibits lung cancer cell growth and migration via calcium/calmodulin-dependent ferroptosis. Signal Transduct Target Ther 2020;5:51.

139 Tang Z, Jiang W, Mao M, et al. Deubiquitinase USP35 modulates ferroptosis in lung cancer via targeting ferroportin. Clin Transl Med 2021;11:e390.

140 Gai C, Yu M, Li Z, et al. Acetaminophen sensitizing erastininduced ferroptosis via modulation of Nrf2/heme oxygenase-1 signaling pathway in non-small-cell lung cancer. J Cell Physiol 2020;235:3329-39.

141 Gai C, Liu C, Wu X, et al. MT1DP loaded by folate-modified liposomes sensitizes erastin-induced ferroptosis via regulating miR365a-3p/NRF2 axis in non-small cell lung cancer cells. Cell Death Dis 2020;11:751.

142 Xia Y, Liu S, Li C, et al. Discovery of a novel ferroptosis inducertalaroconvolutin A-killing colorectal cancer cells in vitro and in vivo. Cell Death Dis 2020;11:988.

143 Zhang L, Liu W, Liu F, et al. Corrigendum to "IMCA Induces Ferroptosis Mediated by SLC7A11 through the AMPK mTOR Pathway in Colorectal Cancer". Oxid Med Cell Longev 2020;2020:1-2.

144 Buccarelli M, Marconi M, Pacioni S, et al. Inhibition of autophagy increases susceptibility of glioblastoma stem cells to temozolomide by igniting ferroptosis. Cell Death Dis 2018;9:841.

145 Chen Y, Li N, Wang H, et al. Amentoflavone suppresses cell proliferation and induces cell death through triggering autophagydependent ferroptosis in human glioma. Life Sci 2020;247:117425.

146 Chen Y, Mi Y, Zhang X, et al. Dihydroartemisinin-induced unfolded protein response feedback attenuates ferroptosis via PERK/ ATF4/HSPA5 pathway in glioma cells. J Exp Clin Cancer Res 2019;38:402. 
147 Wang Z, Ding Y, Wang X, et al. Pseudolaric acid B triggers ferroptosis in glioma cells via activation of Nox4 and inhibition of xCT. Cancer Lett 2018;428:21-33.

148 Zhang Y, Tan H, Daniels JD, et al. Imidazole ketone erastin induces ferroptosis and slows tumor growth in a mouse lymphoma model. Cell Chem Biol 2019;26:623-33.

149 Chan DW, Yung MM, Chan Y-S, et al. Map30 protein from Momordica charantia is therapeutic and has synergic activity with cisplatin against ovarian cancer in vivo by altering metabolism and inducing ferroptosis. Pharmacol Res 2020;161:105157.

150 Tesfay L, Paul BT, Konstorum A, et al. Stearoyl-Coa desaturase 1 protects ovarian cancer cells from Ferroptotic cell death. Cancer Res 2019;79:5355-66.
151 Hong T, Lei G, Chen X, et al. Parp inhibition promotes ferroptosis via repressing Slc7a11 and synergizes with ferroptosis inducers in BRCA-proficient ovarian cancer. Redox Biol 2021;42:10.1016/j. redox.2021.101928.

152 Nagpal A, Redvers RP, Ling X, et al. Neoadjuvant neratinib promotes ferroptosis and inhibits brain metastasis in a novel syngeneic model of spontaneous HER2 $2^{+v e}$ breast cancer metastasis. Breast Cancer Res 2019;21:10.1186/s13058-019-1177-1.

153 Luo M, Wu L, Zhang K, et al. miR-137 regulates ferroptosis by targeting glutamine transporter SLC1A5 in melanoma. Cell Death Differ 2018;25:10.1038/s41418-017-0053-8:1457-72. 